\title{
Testing Slope Homogeneity in Quantile Regression Panel Data with an Application to the Cross-Section of Stock Returns
}

\author{
Antonio F. Galvao* $\quad$ Ted Juhl ${ }^{\dagger}$ Gabriel Montes-Rojas ${ }^{\ddagger} \quad$ Jose Olmo $^{\S}$
}

January 31, 2017

\begin{abstract}
This paper proposes tests for slope homogeneity across individuals in quantile regression fixed effects panel data models. The tests are based on the Swamy statistic. We establish the asymptotic null distribution of the tests under large panels, with sequential and joint limits. A prominent advantage of the proposed tests is that they are easy to implement in empirical applications. Monte Carlo experiments show that the proposed tests have good finite sample performance in terms of size and power. The tests are then applied to study the cross-section of firms' excess asset returns using financial data on U.S. firms. The slope homogeneity tests allow us to assess, for a given quantile of the distribution of excess returns, whether the linear effect of the pricing factors in standard linear asset pricing models is the same across stocks. The results confirm the validity of those models for the mean and central quantiles of the distribution of the firms' excess asset returns. However, for tail quantiles, the slope homogeneity tests reject the null hypothesis providing empirical evidence of pricing anomalies. This suggests that the effect of firm characteristics on the distribution of excess returns is heterogenous across stocks during booms and busts.
\end{abstract}

Keywords: Panel data, quantile regression, slope homogeneity, stock returns.

JEL classification: C12, C23, G10.

${ }^{*}$ Corresponding author: Tippie College of Business, University of Iowa, Department of Economics, W284 PBB, 21 E. Market Street, Iowa City, IA 52242. United States. E-mail: antonio-galvao@uiowa.edu

${ }^{\dagger}$ Department of Economics, University of Kansas, 415 Snow, Lawrence, KS 66045. E-mail: juhl@ku.edu

${ }^{\ddagger}$ CONICET-Universidad de San Andrés, Vito Dumas 284, Victoria, B1644, Provincia de Buenos Aires, Argentina. E-mail: gmontesrojas@udesa.edu.ar

${ }^{\S}$ Department of Economics, University of Southampton, Highfield Lane, Southampton, SO17 1BH. United Kingdom. E-mail: j.b.olmo@soton.ac.uk 


\section{Introduction}

The collection of more data is a welcome feature of modern empirical economics and finance. Given the larger datasets, researchers are able to consider more realistic models that allow for heterogeneity. An important class of statistical models that control for (unobserved) heterogeneity is panel data models. It has become usual in panel data applications to impose a concomitant assumption of heterogeneous individual specific intercepts and homogeneous slope coefficients across individuals. The former condition has become standard in the panel data literature. However, the latter constraint might be seen as excessively strong and has become controversial as the availability of data increases.

Moved by the question on the benefits of pooling vis-à-vis heterogeneous estimators, there is a literature investigating the advantages and disadvantages of estimating the panel data model separately for each cross-sectional unit or by pooling the data (see, e.g., Maddala and Hu (1996), Maddala, Trost, Li, and Joutz (1997), Baltagi, Griffin, and Xiong (2000), and Maddala, Li, and Srivastava (2001)). These models are based on the intuition that homogeneity of the slope coefficients is often an unreasonable assumption, and thus they allow for cross-section heterogeneity. ${ }^{1}$ Hsiao and Sun (2000) argue that if the individuals do not share homogeneous coefficients, fixed effects (FE) estimation may not estimate any parameter of interest and hence, in empirical work, it is important to use formal tests to evaluate the conjecture of homogeneous coefficients across individuals.

There are several tests available in the literature for the hypothesis of slope homogeneity across individuals for average regression models, including, among others, Pesaran, Smith, and Im (1996), Phillips and Sul (2003), Pesaran and Yamagata (2008), Blomquist and Westerlund (2013), and Su and Chen (2013). Pesaran, Smith, and Im (1996) propose an application of the Hausman (1978) procedure where the FE estimator is compared with the mean group estimator. Phillips and Sul (2003) suggest a "Hausman-type" test in the context of stationary first-order autoregressive panel data models, where the cross-section, $n$, is fixed as the time-series, $T$, goes to infinity. Hsiao (2003) describes a variation of the Breusch and Pagan (1979) test, which is valid when both $n$ and $T$ dimensions tend to infinity. More recently, Pesaran and Yamagata (2008) propose a dispersion type test based on Swamy (1970)

\footnotetext{
${ }^{1}$ Another related literature includes random coefficient models. Swamy and Tavlas (2007) and Hsiao and Pesaran (2008) are good surveys for these models. For a general discussion on the modeling of heterogeneity, see Browning and Carro (2007).
} 
type test. They standardize the Swamy type test so that the test can be applied when both $n$ and $T$ are large. Blomquist and Westerlund (2013) develop a test that is robust to general forms of cross-sectional dependence and serial correlation. Su and Chen (2013) propose a test for slope homogeneity in large-dimensional panel data models with interactive FE. Nevertheless, to the best of our knowledge, there is no available test of slope homogeneity for quantile regression $(\mathrm{QR})$ panel data models.

QR is an important tool to analyze heterogeneity in statistical models. Recently, there has been a growing literature on estimation and testing using QR panel data models. These models have provided a valuable method of statistical analysis of the heterogenous effects of policy variables. This is especially true for program evaluation studies in economics and statistics, where these methods help to analyze how treatments or social programs affect the outcome distributions of interest. Koenker (2004) introduces a general approach to estimation of QR models for panel data. Controlling for individual specific heterogeneity via FE while exploring heterogeneous covariate effects within the QR framework offers a flexible approach to the analysis of panel data. ${ }^{2}$

Motivated by the fact that formal tests for homogeneity of the slopes in panel data models are an indispensable tool for practitioners and also by the recent strong influence of QR panel data methods, this paper contributes to the literature by developing testing procedures for homogeneity of the slope coefficients across individuals for FE QR models. Thus, we propose two tests, a Swamy $(\widehat{S})$ and a standardized Swamy $(\widehat{\Delta})$ type tests, with the null hypothesis of slope homogeneity across individuals for a given quantile, $\tau$, of interest. We derive the limiting distributions of the tests under different asymptotic sample size conditions. In particular, we show that under some regularity conditions and the null hypothesis of the homogeneous slope coefficients, $\widehat{S}$ converges to a chi-square distribution as $T \rightarrow \infty$ and $n$ is fixed; and also $\widehat{\Delta}$ converges to the standard normal distribution as both $(T, n) \rightarrow \infty$, sequentially and jointly. When the null hypothesis of homogeneous slope coefficients is rejected for some $\tau$, there is evidence of heterogeneous covariate effects across individuals, and as a result using FE QR assuming homogeneous slope coefficients is inappropriate.

The theoretical results of the paper suggest that the proposed statistics require large

\footnotetext{
${ }^{2}$ For other recent developments in QR panel data, see, e.g., Wei and He (2006), Wang and Fygenson (2009), Canay (2011), Kato, Galvao, and Montes-Rojas (2012), Galvao, Lamarche, and Lima (2013), Chernozhukov, Fernandez-Val, Hahn, and Newey (2013), Galvao and Wang (2015), Arellano and Bonhomme (2016), and Chetverikov, Larsen, and Palmer (2016).
} 
values of $n$ and $T$ for validity. In light of these requirements (as well as the relationship required between $n$ and $T$ ), we perform an extensive Monte Carlo experiment that explores the statistics for a wide variety of sample sizes. We are interested in exploring the finite-sample performance of the test for static and dynamic linear regression models. The simulation results show that the asymptotic distribution is accurate for both processes, with the proposed tests possessing empirical size very close to the nominal size and good power performance even for relatively small values of $T$ compared to $n$.

This novel testing methodology is applied to statistically assess the correct specification of linear asset pricing models for describing the conditional quantile function of the excess returns on risky assets. To do this, and motivated by the theoretical contributions of Rostek (2010), and more specifically Giovannetti (2013), that extends standard linear average asset pricing formulations to the quantile process, we consider a quantile model for the risk premium on risky assets. In this setup, our slope homogeneity tests allow us to assess, for a given quantile of the distribution of excess returns, whether the effect of the pricing factors in standard linear asset pricing models is the same across stocks. By doing so, we extend cross-sectional linear asset pricing formulations for the risk premium to the quantile process. Our approach is more general than the quantile processes obtained from standard locationscale models for asset returns. As a byproduct of our test, under the null hypothesis, our QR panel data model can be interpreted as a linear asset pricing model that accommodates different coefficients across quantiles and that is robust to the presence of asset-specific FE.

In this empirical application, we focus on the recent contributions of Kogan and Papanikolaou $(2013,2014)$ relating firm characteristics and stock returns. These authors propose a linear model to explain the risk premium on a cross-section of U.S. stocks as a function of firm characteristics. We extend this model to the QR case. To do this, we exploit a dataset comprising 294 firms' asset returns that are regressed on a set of risk factors given by a market portfolio excess return and several firm characteristics such as earnings before interest and taxes as a proportion of total assets, market-to-book ratio, obtained as the inverse of the book-to-market ratio, market debt ratio, depreciation as a proportion of total assets, the log of asset size, and a variable that captures the proportion of property, plant, and equipment over total assets.

The results of the empirical exercise do not provide statistical evidence to reject the null hypothesis of homogeneity of the slope parameters for the mean and central quantiles of the 
distribution of the firms' excess asset returns. In particular, the estimates obtained from the panel data regression models are in line with those of Kogan and Papanikolaou (2013) and reveal the statistical significance of firm characteristics for predicting the excess asset returns on U.S. stocks. The results of the tests cast strong doubt, however, on the homogeneity of the sensitivity of these returns to firm characteristics in the tails of the distribution of excess asset returns. These tests are also applied to assess the homogeneity of the marginal effects of specific risk factors on the excess asset returns. In line with the results for the joint slope homogeneity tests, we do not find significant evidence to reject homogeneity in the mean and central quantiles for most of the regression variables acting as risk factors. Nevertheless, we find strong empirical evidence to reject the null hypothesis for the marginal effect of firms' book-to-market ratio. In order to obtain a clearer insight into this result, we divide our sample into value stocks and growth stocks and apply the slope homogeneity testing procedure for each subsample. The test rejects the null hypothesis of slope homogeneity for growth stocks but nor for value stocks. We interpret these results as empirical evidence of the suitability of our empirical panel data equation for pricing more established, value, stocks, but not for pricing riskier and newer, growth, stocks.

The rest of the paper is organized as follows. Section 2 describes and discusses the null hypothesis and the tests proposed. Sections 3 presents the asymptotic properties of the test statistics. The Monte Carlo simulation results are reported in Section 4. In Section 5, we illustrate the new approach with an application to empirical asset pricing, and Section 6 concludes the paper. An appendix contains the mathematical proofs.

\section{The null hypothesis and the proposed tests}

We consider a linear fixed effects (FE) quantile regression (QR) panel model with $n$ individuals and $T$ time periods for each individual as

$$
Q_{y_{i t}}\left(\tau \mid \boldsymbol{x}_{i t}, \alpha_{i 0}(\tau)\right)=\alpha_{i 0}(\tau)+\boldsymbol{x}_{i t}^{\top} \boldsymbol{\beta}_{i 0}(\tau) t=1, \ldots, T ; i=1, \cdots, n
$$

where $y_{i t}$ is the response variable for the $i$-th individual at time $t, \alpha_{i 0}$ is the $i$-th individual effect, $\boldsymbol{x}_{i t}$ is a $k$ dimensional vector of explanatory variables, $Q_{y_{i t}}\left(\tau \mid \boldsymbol{x}_{i t}, \alpha_{i 0}(\tau)\right)$ is the $\tau$ quantile of $y_{i t}$ given $\left(\alpha_{i 0}, \boldsymbol{x}_{i t}\right)$. In general, the coefficients $\alpha_{i 0}(\tau)$ and $\boldsymbol{\beta}(\tau)$ can depend on the quantile index $\tau$. Since $\tau$ is fixed throughout the paper, we will suppress this dependence 
for notational simplicity. We make no parametric assumption on the relationship between $\alpha_{i 0}$ and $\boldsymbol{x}_{i t}$.

For notational simplicity, let $\boldsymbol{X}_{i t}$ be the corresponding covariates with the first element being one, and $\boldsymbol{\theta}_{i 0}:=\left(\alpha_{i 0}, \boldsymbol{\beta}_{i 0}^{\top}\right)^{\top}$ be a $k+1$ vector of coefficients. Thus, model (2.1) can be written concisely as

$$
Q_{y_{i t}}\left(\tau \mid \boldsymbol{X}_{i t}\right)=\boldsymbol{X}_{i t}^{\top} \boldsymbol{\theta}_{i 0}=\alpha_{i 0}+\boldsymbol{x}_{i t}^{\top} \boldsymbol{\beta}_{i 0}
$$

For a given $\tau \in(0,1)$, we wish to test the following hypothesis of slope homogeneity across individuals

$$
H_{0}: \boldsymbol{\beta}_{i 0}=\boldsymbol{\beta}_{0}
$$

for some fixed vector $\boldsymbol{\beta}_{0}$ for all $i$, against the alternatives

$$
H_{1}: \boldsymbol{\beta}_{i 0} \neq \boldsymbol{\beta}_{j 0} \quad \exists i, j
$$

To implement the tests, the strategy is to estimate the QR coefficients using the time series for each individual, and then compare these resulting estimates with $\boldsymbol{\beta}_{0}$. Under the null, the estimates for all individuals should be close to $\boldsymbol{\beta}_{0}$. Therefore, a large deviation of the individual estimates from $\boldsymbol{\beta}_{0}$ indicates that the null should be rejected. However, in general, we do not observe the true coefficients $\boldsymbol{\beta}_{0}$, and thus we replace it with a weighted average of the estimates, $\widehat{\boldsymbol{\beta}}_{i}$, from each individual. Under the null, all the $\widehat{\boldsymbol{\beta}}_{i}$ should be close to each other, and to any weighted average of those estimates.

More specifically, first we estimate $\boldsymbol{\beta}_{i 0}$ for each individual $i$. Denote the slope regression quantile estimate for each individual $i$ by $\widehat{\boldsymbol{\beta}}_{i}:=\Xi \widehat{\boldsymbol{\theta}}_{i}$, with $\Xi:=\left[0_{k \times 1} \mid I_{k \times k}\right]$, and

$$
\widehat{\boldsymbol{\theta}}_{i}:=\underset{\boldsymbol{\theta} \in \mathbb{R}^{k+1}}{\arg \min } \frac{1}{T} \sum_{t=1}^{T} \rho_{\tau}\left(y_{i t}-\boldsymbol{X}_{i t}^{\top} \boldsymbol{\theta}\right),
$$

and $\rho_{\tau}(u):=u(\tau-1\{u \leq 0\})$ as in Koenker and Bassett (1978). Thus, for each individual $i$ regression, the matrix $\Xi$ selects the slope coefficients of interest.

Next, we need to estimate $\boldsymbol{\beta}_{0}$. To do so, we use a FE minimum distance (MD) estimator, which is a weighted average of the slope regression quantiles. Define the following MD estimator

$$
\widehat{\boldsymbol{\beta}}_{M D}=\left(\sum_{i=1}^{n} \widehat{V}_{i}^{-1}\right)^{-1} \sum_{i=1}^{n} \widehat{V}_{i}^{-1} \widehat{\boldsymbol{\beta}}_{i}
$$


with $\widehat{V}_{i}:=\Xi \widehat{\widetilde{V}}_{i} \Xi^{\top}$, where $\widehat{\widetilde{V}}_{i}$ is a consistent estimator of the asymptotic variance-covariance matrix of the individual regression quantiles

$$
\widetilde{V}_{i}:=\tau(1-\tau) \Gamma_{i}^{-1} \Omega_{i} \Gamma_{i}^{-1}
$$

with $\Gamma_{i}:=E\left[f_{i}\left(0 \mid \boldsymbol{X}_{i t}\right) \boldsymbol{X}_{i t} \boldsymbol{X}_{i t}^{\top}\right]$ and $\Omega_{i}:=E\left[\boldsymbol{X}_{i t} \boldsymbol{X}_{i t}^{\top}\right]$ if the data are independent and identically distributed (i.i.d.) within each individual. We discuss the practical estimation of $\widetilde{V}_{i}$ below. The weighted mean of the $\widehat{\boldsymbol{\beta}}_{i}$ defined above is a MD estimator with weights being the inverse of the asymptotic variance-covariance matrices of the slope regression quantiles. Galvao and Wang (2015) propose this MD QR estimator for panel data models with FE and establish its asymptotic properties. For a thorough discussion of the MD estimators, see Newey and McFadden (1994), and Hsiao (2003). This FE MD-QR is the benchmark for the comparison with the $\widehat{\boldsymbol{\beta}}_{i}$ 's in our tests.

To formally test the hypothesis of interest $\left(H_{0}: \boldsymbol{\beta}_{i 0}=\boldsymbol{\beta}_{0}\right)$ we need a metric to compare the estimates $\widehat{\boldsymbol{\beta}}_{i}$ and $\widehat{\boldsymbol{\beta}}_{M D}$. Thus, we propose a $\mathrm{QR}$ version of the Swamy type test as

$$
\widehat{S}(\tau):=\sum_{i=1}^{n}\left(\widehat{\boldsymbol{\beta}}_{i}-\widehat{\boldsymbol{\beta}}_{M D}\right)^{\top}\left(\frac{\widehat{V}_{i}}{T}\right)^{-1}\left(\widehat{\boldsymbol{\beta}}_{i}-\widehat{\boldsymbol{\beta}}_{M D}\right),
$$

and also a standardized Swamy test as

$$
\widehat{\Delta}(\tau)=\sqrt{n}\left(\frac{\frac{1}{n} \widehat{S}(\tau)-k}{\sqrt{2 k}}\right)
$$

Both tests $\widehat{S}(\tau)$ and $\widehat{\Delta}(\tau)$ depend on the quantile index $\tau$, however we suppress this dependence for simplicity. As it will be clear later, when $T$ is large and $n$ is fixed, $\widehat{S}$ is asymptotically $\chi_{(n-1) k}^{2}$ distributed. Therefore, $E\left[\frac{1}{n} \widehat{S}\right]$ is approximately $k$ and $\operatorname{Var}\left[\frac{1}{n} \widehat{S}\right]$ is approximately $\frac{2 k}{n}$. In fact, under some mild conditions, the previous statement holds, and the standardized Swamy test statistic can be approximated by a standard normal random variable.

The intuition behind the tests is that under the null of slope homogeneity, all $\boldsymbol{\beta}_{i 0}$ are the same, and then all estimates $\widehat{\boldsymbol{\beta}}_{i}$ are close to each other and consequently to $\boldsymbol{\beta}_{0}$, which is estimated by the weighted average $\widehat{\boldsymbol{\beta}}_{M D}$. Therefore, both $\widehat{S}$ and $\widehat{\Delta}$ should be small. If there is evidence that either $\widehat{S}$ or $\widehat{\Delta}$ is large, this casts doubts that the null hypothesis of homogeneity of the slope coefficients holds. ${ }^{3}$

\footnotetext{
${ }^{3}$ It is worth noting that we propose the MD estimator $\widehat{\boldsymbol{\beta}}_{M D}$ as the benchmark to construct the test
} 


\section{$3 \quad$ Asymptotic properties of the tests}

In this section, we investigate the asymptotic properties of the proposed tests. The strategy followed is to derive the results under the i.i.d. assumption first, and then extend the results to a processes exhibiting serial dependence, and in particular to a stationary $\beta$-mixing processes, such as those encountered when modeling financial data.

We follow both the slope heterogeneity testing and the panel QR literatures and present results for large panels. In particular, we derive the limiting distribution of the Swamy test $(\widehat{S})$ as $T \rightarrow \infty$, and the limiting distributions of the standardized Swamy test $(\widehat{\Delta})$ under two different asymptotics: $T$ and $n$ tending to infinity sequentially and simultaneously. The sequential asymptotics are defined as $T$ diverging to infinity first, and then $n$. In the definition of the simultaneous asymptotics, $T$ and $n$ tend to infinity at the same time. We do not specify the exact relationship between $n$ and $T$, although we maintain that $T$ depends on $n$. For notational simplicity, we suppress this dependence. For a detailed discussion on sequential and simultaneous asymptotics for panel data, see Phillips and Moon (1999, 2000). In what follows, we adopt the following notation: $(T, n)_{s e q} \rightarrow \infty$ means that first $T \rightarrow \infty$ and then $n \rightarrow \infty$, while $(T, n) \rightarrow \infty$ means $T$ and $n$ tend to infinity simultaneously.

We highlight that the use of large panel data asymptotics is common for testing slope homogeneity in the mean regression literature. The Swamy type test was originally devised for panel data with small $n$ and large $T$, while the standardized Swamy test was developed for panel data with both $n$ and $T$ large. ${ }^{4}$ In this paper, there are two main reasons for requiring large panels. First, we need to consistently estimate $\boldsymbol{\beta}_{i 0}$ for each individual, and the data employed in its estimation relies only on the time dimension. Second, the benchmark parameter, $\boldsymbol{\beta}_{0}$, is estimated by $\widehat{\boldsymbol{\beta}}_{M D}$, which is similar to a FE QR estimator. To achieve consistency of $\widehat{\boldsymbol{\beta}}_{M D}$, it is also required that $T$ tends to infinity. We note that using large panels is standard in panel data FE QR literature (see e.g., Galvao and Wang (2015) and

statistics in this paper. However, one could replace the MD estimator with the standard consistent FE-QR estimator, $\widehat{\boldsymbol{\beta}}_{F E}$, introduced by Koenker (2004). There are a few reasons, though, for employing the MD estimator. First, the use of the MD-QR is analogous to a re-weighted version of the FE estimator for the OLS case and similar to the estimator used in Pesaran and Yamagata (2008). Second, as shown in Galvao and Wang (2015) the MD-QR estimator is efficient in the class of MD estimators, and hence more efficient than the standard FE-QR. Third, the MD estimator simplifies the derivation of the limiting distributions of the test statistics as shown in the proofs.

${ }^{4}$ For example, Phillips and Sul (2003) use $n$ fixed and $T$ diverging to infinity. Hsiao (2003) requires both $n$ and $T$ tending to infinity. Pesaran and Yamagata (2008) also use both $n$ and $T$ large, and more recently, $\mathrm{Su}$ and Chen (2013) and Ando and Bai (2014). 
Kato, Galvao, and Montes-Rojas (2012)). In general, the FE QR estimators are subject to the incidental parameters problem (Neyman and Scott (1948)). Therefore, it is essential to allow $T$ to increase to infinity to achieve consistency and asymptotic normality of the FE QR estimators.

Next, we derive the limiting distribution of the test statistics. To this end, we consider the following set of conditions.

A1. $\left\{\left(y_{i t}, \boldsymbol{X}_{i t}\right)\right\}$ is independent across $i$, and independent and identically distributed (i.i.d.) for each $i$.

A2. There is a constant $M$ such that $\max _{1 \leq t \leq T, 1 \leq i \leq n}\left\|\boldsymbol{X}_{i t}\right\|<M$.

A3. For each $\delta>0$,

$$
\epsilon_{\delta}:=\inf _{1 \leq i \leq n} \inf _{\|\boldsymbol{\theta}\|=\delta} E\left[\int_{0}^{\boldsymbol{X}_{i t}^{\top} \boldsymbol{\theta}}\left\{F_{i}\left(s \mid \boldsymbol{X}_{i t}\right)-\tau\right\} d s\right]>0
$$

where $F_{i}\left(s \mid \boldsymbol{X}_{i t}\right)$ is the distribution function of the innovations conditional on the covariates.

A4. Let $u_{i t}=y_{i t}-\alpha_{i 0}-\boldsymbol{x}_{i t}^{\top} \boldsymbol{\beta}_{i 0}$. The conditional density $f_{i}(u \mid \boldsymbol{X})$ is continuously differentiable for each $\boldsymbol{X}$ and $i$, and let $f_{i}^{(1)}(u \mid \boldsymbol{x}):=\partial f_{i}(u \mid \boldsymbol{x}) / \partial u$. There exist $0<C_{L} \leq C_{U}<\infty$ such that $C_{L} \leq f_{i}(u \mid \boldsymbol{X}) \leq C_{U}$ uniformly over $(u, \boldsymbol{X})$ and $i \geq 1$; and there exists $C_{f}>0$ such that $\left|f_{i}^{(1)}(u \mid \boldsymbol{X})\right| \leq C_{f}$.

A5. There exists $\delta_{\Omega}>0$ such that $\min _{1 \leq i \leq n} \min \operatorname{eig}\left(\Omega_{i}\right) \geq \delta_{\Omega}$.

Condition A1 assumes, for simplicity, that the data are independent across individuals, and i.i.d. within each individual. Nevertheless, below we will also study the asymptotic properties of the tests for the dependent case. Condition A2 assumes that the covariates are uniformly bounded, which is used in Condition A3 of Koenker (2004) and Condition (a) of Theorem 1 of Chernozhukov and Hong (2001). A slightly weaker assumption in Kato, Galvao, and Montes-Rojas (2012) assumes uniform boundedness of the regressors almost surely. In addition, Condition A3 is an identification condition of $\left(\alpha_{i}, \boldsymbol{\beta}_{i 0}\right)$. This condition is usual in the literature. It is the same as A3 of Kato, Galvao, and Montes-Rojas (2012) and corresponds to Condition 3 of Hahn and Newey (2004). The condition states that $\left(\alpha_{i 0}, \boldsymbol{\beta}_{i 0}\right)$ produce $\tau$-th conditional quantile zero. The first three assumptions are used to guarantee 
the uniform consistency of the QR coefficients across individuals. Condition A4 restricts the smoothness and the boundedness of the density (and its derivatives) of the errors conditional on the covariates. This condition is now quite familiar in the QR literature. Condition A5 is concerned with the asymptotic covariance matrix of $\widehat{\boldsymbol{\beta}}_{i}$, as presented above. It assures that $\Omega_{i}^{-1}$ are bounded uniformly across $i$. Also, the uniform upper and lower bounds of the continuous density functions together with Conditions A2 and A5 guarantee that both $\Gamma_{i}$ 's and their inverses are uniformly bounded across $i$. Therefore, the variance-covariance matrices of the regression quantiles $\widetilde{V}_{i}$ and their inverses are uniformly bounded.

In applications, the variance-covariance matrices are unknown and need to be estimated. For sequential asymptotics, we make the following assumption:

A6. $\widehat{V}_{i}=V_{i}+o_{p}(1)$ for each $i$ as $T \rightarrow \infty$.

In the situation when $n$ and $T$ tend to infinity simultaneously, we impose the following condition:

A6'. $\widehat{V}_{i}=V_{i}+O_{p}\left(T^{-1 / 2} h_{n}^{-1 / 2}\right)$ for some $h_{n} \downarrow 0$ uniformly across $i$ and $\lim _{n \rightarrow \infty} \frac{n}{T h_{n}}=0$ as $n \rightarrow \infty$.

An estimator satisfying these conditions is $\widehat{V}_{i}=\Xi \widehat{\widetilde{V}}_{i} \Xi^{\top}$, where

$$
\begin{aligned}
\widehat{\widetilde{V}}_{i} & =\tau(1-\tau) \widehat{\Gamma}_{i}^{-1} \widehat{\Omega}_{i} \widehat{\Gamma}_{i}^{-1}, \\
\widehat{\Gamma}_{i} & =\frac{1}{T} \sum_{t=1}^{T} K_{h_{n}}\left(\widehat{u}_{i t}\right) \boldsymbol{X}_{i t} \boldsymbol{X}_{i t}^{\top}, \\
\widehat{\Omega}_{i} & =\frac{1}{T} \sum_{t=1}^{T} \boldsymbol{X}_{i t} \boldsymbol{X}_{i t}^{\top},
\end{aligned}
$$

$\widehat{u}_{i t}=y_{i t}-\widehat{\alpha}_{i}-\boldsymbol{x}_{i t}^{\top} \widehat{\boldsymbol{\beta}}_{i}$, and $K_{h_{n}}(\cdot)$ defined as in Kato, Galvao, and Montes-Rojas (2012). For a study of the convergence rate of the Powell's kernel estimator, see Kato (2012).

Now we present the asymptotic distributions of the test statistics.

\section{Theorem 1.}

1. Under Conditions A1-A6, we have $\widehat{S} \stackrel{d}{\rightarrow} \chi_{(n-1) k}^{2}$ as $T \rightarrow \infty$ and $n$ is fixed;

2. Under Conditions A1-A6, we have $\widehat{\Delta} \stackrel{d}{\rightarrow} N(0,1)$ as $(T, n)_{s e q} \rightarrow \infty$; 
3. Under Conditions A1-A5, and A6', we have $\widehat{\Delta} \stackrel{d}{\rightarrow} N(0,1)$ as $(T, n) \rightarrow \infty$, provided $\frac{n^{2} \log n}{T}\left|\log \delta_{n}\right|^{2} \rightarrow 0$, where $\delta_{n}=\sqrt{\frac{\log n}{T}}$.

Proof. See Appendix.

It is worth noting the restrictive condition on the times series (i.e. $\frac{n^{2} \log n}{T}\left|\log \delta_{n}\right|^{2} \rightarrow 0$, where $\delta_{n}=\sqrt{\frac{\log n}{T}}$ ) for Theorem 1 part 3. This condition is used only to control the remainder term when establishing the first order approximation of the test statistic. It reflects the fact that the rate of remainder term of the Bahadur representation of the panel data QR estimator is of order $\left(T / \log n^{-3 / 4}\right)$. The slower convergence rate of the remainder term is due to the non-smoothness of the scores. It is important to also note that the growth condition on $T$ for establishing $\sqrt{n T}$-consistency of the MD QR estimator (or other FE estimators in general) is determined so that the remainder term vanishes. Thus, the rate of the remainder term is essential in the asymptotic analysis of the FE estimation when $n$ and $T$ jointly go to infinity.

Now we extend the previous results to consider dependent data such that, within each individual, the data are stationary and $\beta$-mixing. We maintain the independence across individuals as in A1. To accommodate the dependence across time, we follow the literature (see, e.g., Pesaran and Yamagata (2008) and Blomquist and Westerlund (2013)) and adjust the variance-covariance matrices in the corresponding test statistics. In particular, we are interested in testing the same null hypothesis: $H_{0}: \boldsymbol{\beta}_{i 0}=\boldsymbol{\beta}_{0}$ using the Swamy and standardized Swamy tests

$$
\begin{gathered}
\widehat{S}=\sum_{i=1}^{n}\left(\widehat{\boldsymbol{\beta}}_{i}-\widehat{\boldsymbol{\beta}}_{M D}\right)^{\top}\left(\frac{\widehat{V}_{i}}{T}\right)^{-1}\left(\widehat{\boldsymbol{\beta}}_{i}-\widehat{\boldsymbol{\beta}}_{M D}\right) \\
\widehat{\Delta}=\sqrt{n}\left(\frac{\frac{1}{n} \widehat{S}-k}{\sqrt{2 k}}\right) .
\end{gathered}
$$

In this context, we need to estimate $\widehat{V}_{i}$ consistently. We first formally state the conditions for the statistics to have desirable properties.

B1. For each $i,\left\{\left(y_{i t}, \boldsymbol{X}_{i t}\right), t \geq 1\right\}$ is stationary and $\beta$-mixing time series with $\beta$-mixing coefficient $\beta_{i}(j)$. There exist constants $a \in(0,1)$ and $B>0$ such that $\sup _{i \geq 1} \beta_{i}(j) \leq$ $B a^{j}$ for all $j \geq 1$. 
B2. $f_{i, j}\left(u_{1}, u_{1+j} \mid \boldsymbol{X}_{1}, \boldsymbol{X}_{1+j}\right)$ is uniformly bounded with respect to all the four variables, where $f_{i, j}\left(u_{1}, u_{1+j} \mid \boldsymbol{X}_{1}, \boldsymbol{X}_{1+j}\right)$ is the conditional density of $\left(u_{1}, u_{1+j}\right)$ given $\left(\boldsymbol{X}_{i, 1}, \boldsymbol{X}_{i, 1+j}\right)=$ $\left(\boldsymbol{X}_{1}, \boldsymbol{X}_{1+j}\right)$.

Condition B1 relaxes the assumption of $i . i . d$. within each individual to that of stationary $\beta$-mixing and is similar to Hahn and Kuersteiner (2011). Condition B2 is needed because the data are not i.i.d. and we need to impose a condition on the joint distributions.

The following theorem collects the asymptotic results for stationary $\beta$-mixing data, and provides respective extensions of Theorem 1.

\section{Theorem 2.}

1. Under Conditions $A 2-A 6$ and B1-B2, we have $\widehat{S} \stackrel{d}{\rightarrow} \chi_{(n-1) k}^{2}$ as $T \rightarrow \infty$ and $n$ is fixed;

2. Under Conditions $A 2-A 6$ and B1-B2, we have $\widehat{\Delta} \stackrel{d}{\rightarrow} N(0,1)$ as $(T, n)_{\text {seq }} \rightarrow \infty$;

3. Under Conditions A2-A5, A6', and B1-B2, we have $\widehat{\Delta} \stackrel{d}{\rightarrow} N(0,1)$ as $(T, n) \rightarrow \infty$, provided $\frac{n^{2} \log n}{T}\left|\log \delta_{n}\right|^{2} \rightarrow 0$, where $\delta_{n}=\sqrt{\frac{\log n}{T}}$.

Proof. See Appendix.

The results in Theorem 2 require condition A6 or A6' to hold for stationary and $\beta$-mixing data. In the same fashion, an example of the estimator is $\widehat{V}_{i}=\Xi \widehat{\widetilde{V}}_{i} \Xi^{\top}$, where

$\widehat{\widetilde{V}}_{i}=\widehat{\Gamma}_{i}^{-1} \widehat{\Omega}_{i} \widehat{\Gamma}_{i}^{-1}$,

$\widehat{\Gamma}_{i}=\frac{1}{T} \sum_{t=1}^{T} K_{h_{n}}\left(\widehat{u}_{i t}\right) \boldsymbol{X}_{i t} \boldsymbol{X}_{i t}^{\top}$,

$\widehat{\Omega}_{i}=\frac{\tau(1-\tau)}{T} \sum_{t=1}^{T} \boldsymbol{X}_{i t} \boldsymbol{X}_{i t}^{\top}+\sum_{1 \leq|j| \leq m_{n}}\left(1-\frac{|j|}{T}\right)\left[\frac{1}{T} \sum_{t=\max \{1,-j+1\}}^{\min \{T, T-j\}}\left\{\tau-1\left(\widehat{u}_{i t} \leq 0\right)\right\}\left\{\tau-1\left(\widehat{u}_{i, t+j} \leq 0\right)\right\} \boldsymbol{X}_{i t} \boldsymbol{X}_{i t}^{\top}\right]$,

as discussed in Remark 3.2 in the proof of Theorem 3.2 of Galvao and Kato (2016). Kato (2012) also studies the rate of convergence of the Powell's kernel estimator for stationary $\beta$ mixing data. For an alternative estimator satisfying conditions A6 and A6' with dependent data see Yoon and Galvao (2014). They develop cluster robust inference methods for panel QR models with individual FE and serial correlation. 


\section{$4 \quad$ Finite sample simulations}

In this section, we investigate the finite sample properties of both Swamy $(\widehat{S})$ and standardized Swamy $(\widehat{\Delta})$ tests proposed above. We report empirical size and empirical power at $5 \%$ nominal level of significance for various pairs of $n$ and $T$. The simulations consider sample sizes given by $n \in\{100,200\}$ and $T \in\{50,100\}$. Of particular interest is the case where $T$ is smaller than $n$, and more specifically, the case $T=50, n=200$, that corresponds to our empirical application. We investigate the finite sample properties of the tests for quantiles $\tau \in\{0.10,0.25,0.50,0.75,0.90\}$. The number of replications is 1,000 in each case.

As discussed in the previous sections, the computation of the variance-covariance matrix is a very important step when computing both $\widehat{S}$ and $\widehat{\Delta}$ tests. Thus, to study the impact of the estimation of the variance-covariance matrix in the tests, we report results for two different cases. In particular we consider the Bofinger and the Hall-Sheather bandwidth rules using the bandwidth.rq function in $\mathrm{R}$ for every $\tau .^{5}$

In the following tables, for any sample size combination, we present results for the empirical size (top panels) and empirical power (bottom panels). We collect results for the Swamy type $(\widehat{S})$ test and standardized Swamy $(\widehat{\Delta})$ test for each case.

\subsection{Static model}

The static models under study are the location and location-scale models given by the data generating process

$$
y_{i t}=\alpha_{i}+\beta_{i} x_{i t}+\left(1+\gamma x_{i t}\right) u_{i t}
$$

for $t=1, \cdots, T$ and $i=1, \cdots, n$. The location model has $\gamma=0$, and the locationscale model considers $\gamma=0.1$. The innovations $u_{i t}$ are $i . i . d$. standard normal $\mathcal{N}(0,1)$, and $x_{i t}=0.3 \alpha_{i}+z_{i t}$, where $z_{i t} \stackrel{\text { i.i.d. }}{\sim} \chi_{3}^{2}$. In the simulations, we set $\alpha_{i}=\frac{i-1}{n-1}$ (which means that $\alpha_{i}$ are uniformly distributed in the unit interval, and are fixed for different replications as in Pesaran and Yamagata (2008)).

We would like to test the null hypothesis $H_{0}: \beta_{i}=\beta_{j}$ for all $i, j \in\{1, \cdots, n\}$ against

\footnotetext{
${ }^{5}$ The Bofinger bandwidth is: $h_{n}=n^{1 / 5}\left[4.5^{2} s(t) /\left(s^{\prime \prime}(t)\right)^{2}\right]^{1 / 5}$, where $s / s^{\prime \prime}=f^{2} /\left[2\left(f^{\prime} / f\right)^{2}+\left(f^{\prime} / f-f^{\prime \prime} / f\right)\right]$ with $f=\phi$ and $\left(f^{\prime} / f\right)\left(F^{-1}(t)\right)=\Phi^{-1}(t)$. The Hall-Sheather bandwidth is: $h_{n}=n^{-1 / 3} z_{\alpha}^{2 / 3}\left[1.5 s(t) / s^{\prime \prime}(t)\right]^{1 / 3}$ with $z_{\alpha}$ satisfies $\Phi\left(z_{\alpha}\right)=1-\alpha / 2$ and $\alpha$ denotes the size of the test.
} 
$H_{1}: \beta_{i} \neq \beta_{j}$ for any $i \neq j$. Under the null, we set $\beta_{i}=1$ for all $i$; under the alternative $\beta_{i}=0.25+\frac{i-1}{n / 2-1}$ for $i \leq \frac{n}{2}$ and 1 otherwise.

We first investigate the location model $(\gamma=0)$. Note that for this case, for each $i$, the corresponding slopes are $\beta_{i}(\tau)=\beta_{i}$, i.e. all QR coefficients are equal to the same intra-firm slope coefficient. Furthermore, under the null hypothesis, $\beta_{i}(\tau)=\beta, \forall i$. Table 1 displays the results for both $\widehat{S}$ and $\widehat{\Delta}$. For all quantiles, the empirical sizes are close to the nominal $5 \%$ significance level, and in particular, for $T=50$ sample sizes which correspond to the empirical application. Note however that the size improves as $T$ increases but there is no observed pattern with respect to $n$. The empirical power is 1.0 in all cases showing that the tests are responsive to slope heterogeneity.

\section{[INSERT TABLE 1 HERE]}

We then consider the location-scale model $(\gamma=0.1)$. In this case, for each $i$, the corresponding slopes are $\beta_{i}(\tau)=\beta_{i}+0.1 \Phi^{-1}(\tau)$, i.e. QR coefficients vary across $\tau$. Furthermore, under the null hypothesis, $\beta_{i}(\tau)=\beta(\tau), \forall i$. As such, parameter heterogeneity could be the result of two different things: first, differences in $\beta_{i}$, second, differences in $u_{i t}$. For our purposes, our model concentrates on the first type of heterogeneity. Table 2 collects the results for this location-scale model. As with the previous case, the empirical size is approximately correct for all sample sizes considered but improves as $T$ increases. Empirical power is again 1.0 for all cases.

\section{[INSERT TABLE 2 HERE]}

\subsection{Dynamic model}

In this subsection, we investigate the finite sample properties of the tests for a dynamic autoregressive panel model. The model under study is ${ }^{6}$

$$
y_{i t}=\alpha_{i}+\delta y_{i t-1}+\beta_{i} x_{i t}+u_{i t}
$$

for $t=1, \ldots, T$ and $i=1, \ldots, n$. We follow the set-up in the static model with $u_{i t}$ i.i.d. standard normal, $x_{i t}=0.3 \alpha_{i}+z_{i t}$, where $z_{i t} \stackrel{\text { i.i.d. }}{\sim} \chi_{3}^{2}$, and $\alpha_{i}=\frac{i-1}{n-1}$. In generating $y_{i t}$ we set

\footnotetext{
${ }^{6}$ We are grateful to an anonymous referee for suggesting the specific form of this experiment.
} 
$y_{i,-49}=0$ and discard the first 50 observations, using the observations $t=1$ through $T$ for estimation.

We would like to test the null hypothesis of the static model, i.e. $H_{0}: \beta_{i}=\beta_{j}$ for all $i, j$ against $H_{1}: \beta_{i} \neq \beta_{j}$ for any $i \neq j$, and we have the same null and alternative hypotheses as in the previous experiment. However we consider different persistence parameters $\delta \in$ $\{0.5,0.95\}$ in order to evaluate the tests performance in dynamic autoregressive panels.

Tables 3 summarizes the empirical size and power. The simulations indicate that the tests have empirical size below $5 \%$ in most cases. In general the size performance is better for $\delta=0.50$ than for $\delta=0.95$. Power, on the other hand, increases as both $T$ and $n$ grow. The power is higher for moderate persistence $\delta=0.50$ than for high persistence $\delta=0.95$. The rejection rates are higher at the tails than in the central quantiles. Overall the simulations show that the tests have good performance when the data generating process is a dynamic autoregressive panel.

\section{[INSERT TABLE 3 HERE]}

\section{Asset pricing models using firm characteristics}

Recently, in a search for modeling and capturing heterogeneity, quantiles have become central in economics, finance, and econometrics. From a theoretical perspective, there have been developments on quantile objective functions and asset pricing. Manski (1988) and Rostek (2010) develop the decision-theoretic attributes of quantile maximization and examine risk preferences of quantile maximizers. Following these seminal contributions, Giovannetti (2013) presents a novel extension of the static decision-theoretical framework axiomatized by Rostek (2010) for a dynamic asset pricing setting. In a two-period standard economy with one risky and one risk-free asset, this author derives an arbitrage-free asset pricing model. In this model, the equity premium is no longer based on the covariance between the risky return and the consumption growth. Instead, it is a linear function of the risky

return standard deviation. Empirically, Bassett, Koenker, and Kordas (2004) show that a general form of pessimistic optimization based on Choquet approach may be formulated as a problem of linear QR.

In a similar spirit to Giovannetti (2013) and Bassett, Koenker, and Kordas (2004), we 
propose the following linear asset pricing equation based on no-arbitrage arguments:

$$
Q_{r_{i, t+1}^{e}}\left(\tau \mid \boldsymbol{x}_{i t}, \alpha_{i}(\tau)\right)=\alpha_{i}(\tau)+\boldsymbol{x}_{i t}^{\top} \boldsymbol{\beta}_{i}(\tau) i=1, \cdots, n ; t=1, \ldots, T
$$

with $\tau \in(0,1)$. This function models the quantiles of the excess asset returns conditional on the set of pricing factors $\boldsymbol{x}_{i t}$ and the coefficients $\beta_{i 0}(\tau)$. The latter parameters capture the marginal effects of the pricing factors on the $\tau$ th quantile of $r_{i, t+1}^{e}$. We make a few remarks. First, as for the conditional expected value, the asset pricing formulation holds for the quantile process $(5.1)$ if the vector $\boldsymbol{\beta}_{i}(\tau)$ is the same across assets. This condition can be interpreted as evidence of a common stochastic discount factor for pricing the crosssection of risky assets, see Harrison and Kreps (1979) for the mean process, and entails, under some additional restrictions, the absence of arbitrage opportunities. In this way, our slope homogeneity test can be considered as a statistical device to empirically assess whether the asset pricing specification (5.1) holds for different quantiles of the distribution of excess asset returns. Second, the coefficients $\boldsymbol{\beta}_{i}(\tau)$ may vary across the quantiles implying that the marginal effect of $\boldsymbol{\beta}_{i}(\tau)$ on the excess returns may vary along the conditional distribution of $r_{i, t+1}^{e}$. Finally, it is important to notice that in our context, characterized by firm characteristics as risk factors, and unlike in Fama and French (1993, 2015), the intercept of the pricing equation is not required to be zero. This feature is easily accommodated by our slope homogeneity test that makes allowance for the presence of $\mathrm{FE}, \alpha_{i}(\tau)$, that are stock and quantile specific.

The empirical representation of the quantile pricing equation (5.1) based on panel data analysis is

$$
r_{i, t+1}^{e}=\alpha_{i}(\tau)+\boldsymbol{x}_{i t}^{\top} \boldsymbol{\beta}_{i}(\tau)+\varepsilon_{i, t+1}
$$

where the innovation term $\varepsilon_{i, t+1}$ satisfies $Q_{\tau}\left[\varepsilon_{i, t+1} \mid \alpha_{i}(\tau), \boldsymbol{x}_{i t}\right]=0$. The relevant set of slope homogeneity tests, indexed by $\tau$, is

$$
H_{0}(\tau): \boldsymbol{\beta}_{10}(\tau)=\ldots=\boldsymbol{\beta}_{n 0}(\tau)=\boldsymbol{\beta}_{0}(\tau)
$$

In this application, we apply this test to assess the empirical validity of the asset pricing model proposed by Kogan and Papanikolaou (2013, 2014). These authors consider firmspecific characteristics as the risk factors that describe the risk premium on the cross-section of stock returns. In recent years, this approach has gained support due to the strong evidence of co-movement in stock returns of firms with similar characteristics that is unrelated to their 
exposures to the market portfolio. These empirical patterns are also motivated by the failure of existing models to rationalize the co-movement in returns resulting from sorting firms on firm characteristics. Kogan and Papanikolaou (2014), for example, provide a theoretical framework to study empirical return patterns associated with firm characteristics related to growth opportunities.

The panel of U.S. firms is obtained from Compustat Industrial dataset. The sample consists of annual CRSP/Compustat data from the years 1970 through 2011. Following standard practice, we exclude financial firms (SIC codes 6000-6999), regulated utilities (SIC codes 4900-4999), and non-profit organizations (SIC codes greater than or equal to 9000). We omit firm-years with a missing or negative value for fixed assets and sales, with a missing or less than ten million 1983 dollar book value of total assets, and with growth rates of fixed assets, sales, and book value of total assets greater than 100\%. The log of total assets is the one variable that is not a ratio, and is deflated to the 1983 dollar with the consumer price index obtained from the Bureau of Labor Statistics. The final sample includes a balanced panel of 297 firms with 42 years of data. Three firms are dropped from our original sample due to a lack of convergence for quantile regression (QR) models, implying that $n=294$. In addition, the predictive character of the empirical pricing equations implies that $T=41$. The firms' excess returns are the annual excess return on assets computed over the annual interest rate offered by one-month U.S. Treasury bills.

Although there is no consensus in the literature on the length of the time dimension, we acknowledge that the time dimension selection criteria might favor larger and more mature companies, which may lead to the results being valid only for large and mature companies. However, the average estimated effects from our sample are in line with the consensus in literature, and thus, the results could be applied to all companies. Summary statistics of our variables are provided in Table 4 .

\section{[INSERT TABLE 4 HERE]}

We work with three different model specifications of the vector of factors $\boldsymbol{x}_{i t}$ in (5.2), defined as three-, six- and seven-variable specifications. The first case, three-variable specification, corresponds to a model that considers the pricing factors discussed in Fama and French (1992). That is, we entertain a market portfolio return, MKT and two more covariates given by the firm's market-to-book ratio, denoted as $M B$, and the log of the firm's asset 
size, denoted as LNTA. The second model, six-variable specification, only entertains firmspecific characteristics. Thus, as a measure of profitability we consider $M B$ and $L N T A$ as above, and add earnings before interest and taxes as a proportion of total assets, denoted as EBITTA; market debt ratio, defined as book value of debt over market value of assets and denoted as $M D R$ (to capture firms' leverage); depreciation as a proportion of total assets, denoted as DEPTA; and a variable that captures the proportion of property, plant, and equipment over total assets, FATA. The third model, seven-variable specification, extends the second model by including the market portfolio return $M K T$ as an additional regressor.

\subsection{Three-variable specification}

Consider first the three-variable specification proposed by Fama and French (1992). The first two columns of Table 5 present the evaluation of the joint hypothesis of equal slopes for all three covariates. The table reports the p-values of the $S$ and $\Delta$ tests. The null hypothesis is not rejected for central quantiles and the mean parameter vector. ${ }^{7}$ These findings reveal the existence of a common set of parameters for predicting the risk premium in central quantiles of the distribution of firms' excess asset returns, and hence, are informative about the risk premium on the cross-section of risky assets. The null hypothesis of slope homogeneity is rejected for extreme quantiles of the distribution of excess returns. In the context of Fama and French (1996) and Kogan and Papanikolaou (2013), this result suggests that the factors that capture firm-specific characteristics are an adequate description of the risk premium for the mean and central quantiles but not for the tails of the distribution of excess returns on common stocks. The differences observed in the slope coefficients of the panel data regression models for extreme quantiles suggest that it is not possible to pool the information on the cross-section and time-series dimensions. We interpret this as empirical evidence that rejects the possibility of using firm characteristics as pricing factors for the tail quantiles of the distribution of firms' stock returns.

To add robustness to our analysis and gather more empirical evidence on the predictive power of different firm characteristics, we also perform a marginal analysis of slope homogeneity for each firm characteristic. The p-values for the homogeneity of the slope for the marginal tests, reported in Columns 3 to 8 of Table 5 , exhibit patterns very similar to those

\footnotetext{
${ }^{7}$ For the mean parameter vector we apply the version of the Swamy type test obtained from the mean minimum distance estimator fully developed in Pesaran (2006).
} 
discussed for the joint test. More specifically, these marginal tests provide empirical evidence to reject the null hypothesis in the tails of the distribution but not in the central quantiles. Interestingly, this robustness analysis uncovers the existence of heterogeneity in the effect of the firm's market-to-book ratio on the risk premium of risky assets.

To understand this finding in more detail and to obtain a better insight into the effect of firm characteristics on the risk premium, we divide the sample into two subsamples according to the average value of the market-to-book ratio across firms. This sample split allows us to classify firms into value stocks and growth stocks. More specifically, we classify as value stocks those firms with market-to-book ratio below the sample mean. In contrast, growth stocks are those with market-to-book ratio above the sample mean ${ }^{8}$. In particular, Table 6 reports the p-values of the slope homogeneity tests for value stocks, and Table 7 reports the p-values of the tests for growth stocks.

Interestingly, the results of the test for each subsample reveal differences on the effect of the financial ratio on predicting the stock excess return. Growth stocks exhibit an idiosyncratic slope coefficient for each asset. In contrast, we cannot reject the null hypothesis of slope homogeneity for value stocks. We can interpret these results as empirical evidence of pricing differences between more established stocks, value stocks, that can be priced homogeneously by our asset pricing model, and newer, smaller, riskier stocks, categorized as growth stocks, that reject the null hypothesis of slope homogeneity, and with it the empirical suitability of our panel data asset pricing model.

\section{[INSERT TABLES 5-8 HERE]}

The absence of statistical evidence to reject the null hypothesis of slope homogeneity for the mean and central quantiles allows us to pool the cross-section and time-series dimensions to estimate the empirical three-factor specification using the panel of observations. Table 8 presents OLS and QR estimates of the linear specification under the presence of FE. The results provide overwhelming evidence of the significance of the covariates in predicting the risk premium on the cross-section of asset returns. The sign of the parameters reveals interesting findings on the relationship between firm characteristics and excess asset returns.

\footnotetext{
${ }^{8}$ We could have pursued alternative classifications of firms into value stocks and growth stocks based on other percentiles of the distribution of the market-to-book ratio, as for example, the bottom (top) three deciles of the distribution of firms. Instead, we prefer considering the whole sample of firms in our analysis.
} 
More specifically, firms' $M B$ has a negative effect on the risk premium. This finding is consistent across quantiles and suggests that investors require a higher risk premium as the firms' market-to-book value decreases. Interestingly, for high quantiles of the distribution of asset returns, the value of this financial ratio does not have a statistical effect on the risk premium. Note, however, that for tail quantiles the slope homogeneity test is rejected invalidating model estimates for these quantiles obtained from pooling the data across stocks.

The analysis of the effect of firms' asset size on the risk premium of the cross-section also provides interesting insights. In this case, we observe a positive relationship between risk premium and asset size. This empirical finding is similar to those reported by Fama and MacBeth (1973) and Fama and French (1992). Table 8 also reveals a small but statistically significant effect of the market portfolio return on the risk premium on risky assets. Interestingly, the sign of the slope parameter for this market factor becomes negative for the highest quantiles of the distribution of excess asset returns and suggests the decoupling between the market portfolio return and firms' asset returns for very large firms' returns. Nevertheless, as noted above by the $\mathrm{QR}$ slope homogeneity test, the sensitivity of asset returns to the pricing factors is different for each firm in the tails of the distribution casting doubts on the reliability of the pooled estimates for the tail quantiles.

\subsection{Six-variable specification}

Consider now the econometric specification solely characterized by the above six firm characteristics. The first two columns of Table 9 present the p-values of the joint slope homogeneity tests $S$ and $\Delta$ for the mean and quantile processes. The results are the same as in the previous specification. The slope homogeneity tests do not reject the null hypothesis for the mean and central quantiles but report overwhelming evidence that rejects the null hypothesis in the tails of the distribution of firms' asset returns.

Columns 3 to 14 of Table 9 provide the p-values of the marginal slope homogeneity tests for each firm characteristic. The p-value of the market-to-book variable is 0.019 and suggests that the sensitivity of the firms' excess asset returns to variation of this financial ratio is different across stocks. These findings are confirmed by the results of the tests for the subsamples determined by the mean value of the $M B$ financial ratio but are not reported for the sake of space. As in the preceding exercise, the marginal slope homogeneity test for the 
$M B$ ratio fails to reject the null hypothesis for value stocks but rejects it for growth stocks.

\section{[INSERT TABLES 9-10 HERE]}

Table 10 presents the OLS and QR parameter estimates with FE. The results are qualitatively similar to the previous specification, in particular, the sign of the $M B$ and $L N T A$ slope parameters is the same of the previous specification. The model also includes a profitability measure, EBITTA, a depreciation variable, DEPTA, a variable that captures the proportion of physical assets, FATA, and a variable that measures the firm's leverage, $M D R$. The parameter estimates indicate a negative effect of firms' profitability on the excess asset return. The magnitude of this effect is large and statistically significant across quantiles. The proportion of physical assets over total assets also has a positive and significant effect on the cross-section of asset returns. Interestingly, the leverage of the firm, $M D R$, defined as debt over equity, also has a positive effect on the excess asset return. Firms' depreciation as a proportion of total assets has, on the other hand, a negative effect on the risk premium. It is worth noting that the model estimates for the tail quantiles obtained from pooling the data across stocks are not supported by the QR slope homogeneity tests.

\subsection{Seven-variable specification}

This last specification extends the previous model by also including $M K T$. The empirical findings are consistent across models. It is worth noting that the marginal slope homogeneity test associated with the covariate given by the market portfolio return rejects only the null hypothesis for the higher quantiles of the distribution and not for the lower quantiles as do the rest of covariates. As in the two previous specifications, the slope homogeneity test presents some anomalies associated to market-to-book value, in particular, Table 11 reports a p-value of 0.024 and 0.020 for the test statistics $S$ and $\Delta$, respectively.

\section{[INSERT TABLES 11-12 HERE]}

The estimates of the model parameters for this specification, reported in Table 12, are also very similar to previous specifications. The parameter associated with the market portfolio excess return is small, compared to the magnitude of the rest of model parameters, but statistically significant, helping in the prediction of the risk premium. 


\subsection{Discussion of results}

The absence of statistical evidence to reject the null hypothesis of slope homogeneity validates the existence of an empirical asset pricing equation to price the risk premium of the crosssection of firms' stocks at the mean and central quantiles. This powerful result does not hold, however, for the tail quantiles of the distribution of firms' excess returns. In these cases the slope homogeneity test is rejected suggesting that the effect of firm characteristics is heterogenous across stocks.

A simple interpretation of the rejection of the null hypothesis under our QR estimation methodology for panel data is to consider that excess asset returns in the tails correspond to distress and boom periods. In these periods the heterogeneity in the risk premium of risky assets is produced by investors' overreaction, which produces the underpricing of distressed stocks (see Lakonishok, Shleifer, and Vishny (1994), MacKinlay (1995) and Fama and French (1996)), and overpricing of boom stocks. Risk and reward are driven in these cases by individual circumstances and not by a common asset pricing model.

An alternative interpretation of the rejection of the slope homogeneity hypothesis in the lower and upper quantiles of the distribution of the excess asset returns is due to misspecification of the variance of the test statistics. QR models have higher variance for extreme quantiles as compared to central quantiles, and thus, we could be capturing this effect rather than firm heterogeneity. In order to investigate this issue Figure 1 reports the distribution of the estimated firm-specific coefficients for all quantiles, together with the mean-based estimates. More specifically, each chart reports, for each $\tau$-quantile and the OLS model, the $0.05,0.10,0.20,0.80,0.90$ ad 0.95 percentiles of the firm-specific coefficients. These charts provide empirical evidence suggesting that slope heterogeneity increases in the tail quantiles, with a larger variability of the parameter estimates across stocks in the upper quantiles than in the lower quantiles.

\section{[INSERT FIGURE 1 HERE]}

\section{Conclusion}

We have proposed Swamy and standardized Swamy tests for the null hypothesis of slope homogeneity in quantile regression $(\mathrm{QR})$ panel data fixed effects (FE) models. These tests are 
important tools for practitioners, since they allow researchers to investigate the poolability of individual slopes in the FE-QR framework. We have derived the limiting distribution of the tests for large panels under sequential and joint asymptotics. The interpretation of the test results is simple. If the tests do not reject the null hypothesis of slope homogeneity across individuals, one could estimate the standard FE-QR model. On the other hand, if the tests reject the null hypothesis, one should estimate the parameters of interest using data separately for each individual; for instance, estimating individual QR using time series data.

These tests were applied to assess statistically the validity of linear asset pricing specifications for the quantile of excess asset returns in cross-sectional settings. Our application to a cross-section of U.S. firms reveals the empirical suitability of firm characteristics such as earnings, leverage, asset size, debt, amongst a few others, as pricing factors. For central quantiles, the sensitivity of excess asset returns to variation in firm characteristics is homogeneous across stocks and allows us to interpret our time series prediction model as a panel data pricing equation. However, for tail quantiles, the slope homogeneity test rejects the null hypothesis. This result is interpreted as evidence of pricing anomalies that suggest that the sensitivity of returns to firm characteristics is different across stocks during booms and busts. 


\section{References}

Ando, T., And J. BAI (2014): "A Simple New Test for Slope Homogeniety in Panel Data Models with Interactive Effects," MPRA Paper No. 60795.

Arellano, M., and S. Bonhomme (2016): "Nonlinear Panel Data Estimation via Quantile Regressions," Econometrics Journal, forthcoming.

Baltagi, B., J. Griffin, And W. Xiong (2000): "To Pool or Not to Pool: Homogeneous versus Heterogeneous Estimators Applied to Cigarette Demand," The Review of Economics and Statistics, 82, 117-126.

Bassett, G. W., R. Koenker, and G. Kordas (2004): "Pessimistic Portfolio Allocation and Choquet Expected Utility," Journal of Financial Econometrics, 2, 477-492.

Blomquist, J., And J. Westerlund (2013): “Testing Slope Homogeneity in Large Panels with Serial Correlation," Economics Letters, 121, 374-378.

Breusch, T., and A. Pagan (1979): "A Simple Test for Heteroskedasticity and Random Coefficient Variation," Econometrica, 47, 1287-1294.

Browning, M., and J. Carro (2007): "Heterogeneity and Microeconometrics Modeling," in Advances in Economics and Econometrics, Theory and Applications, Ninth World Congress, Volume III, ed. by R. Blundell, W. Newey, and T. Persson, chap. 3, pp. 47-74. Cambridge Press.

Canay, I. (2011): "A Simple Approach to Quantile Regression for Panel Data," Econometrics Journal, 14, 368-386.

Chernozhukov, V., I. Fernandez-Val, J. Hahn, and W. Newey (2013): "Average and Quantile Effects in Nonseparable Panel Models," Econometrica, 81, 535-580.

Chernozhukov, V., And H. Hong (2001): "Three-Step Censored Quantile Regression and Extramarital Affairs," Journal of the American Statistical Association, 97, 872-882.

Chetverikov, D., B. Larsen, and C. Palmer (2016): "IV Quantile Regression for Group-Level Treatments, with an Application to the Effects of Trade on the Distribution of Wages," Econometrica, 84, 809-833. 
FAma, E. F., And K. R. French (1992): "The Cross-Section of Expected Stock Returns," The Journal of Finance, 67, 427-465.

- (1993): "Common Risk Factors in the Returns on Stocks and Bonds," Journal of Financial Economics, 33, 3-56.

- (1996): "Multifactor Explanations of Asset Pricing Anomalies," The Journal of Finance, 51, 55-84.

(2015): “A Five-Factor Asset Pricing Model," Journal of Financial Economics, $116,1-22$.

Fama, E. F., And J. D. MaсBeth (1973): "Risk, Return, and Equilibrium: Empirical Tests," Journal of Political Economy, 81, 607-636.

Galvao, A. F., And K. Kato (2016): "Smoothed Quantile Regression for Panel Data," Journal of Econometrics, 193, 92-112.

GalvaO, A. F., C. Lamarche, and L. Lima (2013): "Estimation of Censored Quantile Regression for Panel Data with Fixed Effects," Journal of the American Statistical Association, 108, 1075-1089.

GalvaO, A. F., and L. Wang (2015): "Efficient Minimum Distance Estimator for Quantile Regression Fixed Effects Panel Data," Journal of Multivariate Analysis, 133, 1-26.

Giovannetti, B. C. (2013): "Asset Pricing under Quantile Utility Maximization," Review of Financial Economics, 22, 169-179.

Hahn, J., And G. Kuersteiner (2011): "Bias Reduction for Dynamic Nonlinear Panel Model with Fixed Effects," Econometric Theory, DOI: 10.1017/S0266466611000028.

Hahn, J., And W. Newey (2004): "Jackknife and Analytical Bias Reduction for Nonlinear Panel Models," Econometrica, 72, 1295-1319.

Harrison, J., And D. Kreps (1979): "Martingales and Arbitrage in Multiperiod Securities Markets," Journal of Economic Theory, 20, 381-408.

Hausman, J. (1978): "Specification Tests in Econometrics," Econometrica, 46, 1251-1271. 
Hsiao, C. (2003): Analysis of Panel Data. Cambridge University Press, New York, New York.

Hsiao, C., And M. H. Pesaran (2008): "Random Coefficient Models," in Advanced Studies in Theoretical and Applied Econometrics, Vol. 46, The Econometrics of Panel Data: Fundamentals and Recent Developments in Theory and Practice, ed. by L. Matyas, and P. Sevestre. Springer-Verlag, Berlin.

HsiaO, C., And B. Sun (2000): "To Pool or Not to Pool Panel Data," in Penal Data Econometrics: Future Directions: Papers in Honor of Professor Pietro Balestra, ed. by J. Krishnakumar, and E. Ronchetti, pp. 181-198. Elsevier Science.

Kato, K. (2012): "Asymptotic Normality of Powell's Kernel Estimator," Annals of the Institute of Statistical Mathematics, 64, 255-273.

Kato, K., A. F. Galvao, and G. Montes-Rojas (2012): "Asymptotics for Panel Quantile Regression Models with Individual Effects," Journal of Econometrics, 170, 76-91.

Koenker, R. (2004): "Quantile Regression for Longitudinal Data," Journal of Multivariate Analysis, 91, 74-89.

(2005): Quantile Regression. Cambridge University Press, New York, New York.

Koenker, R., And G. W. Bassett (1978): "Regression Quantiles," Econometrica, 46, $33-49$.

Kogan, L., and D. Papanikolaou (2013): "Firm Characteristics and Stock Returns: The Role of Investment-Specific Shocks," Review of Financial Studies, 26, 2718-2759.

- (2014): "Growth Opportunities, Technology Shocks and Asset Prices," The Journal of Finance, 69, 675-718.

Lakonishok, J., A. Shleifer, and R. Vishny (1994): "Contrarian Investment, Extrapolation, and Risk," The Journal of Finance, 49, 1541-1578.

MacKinlay, A. (1995): "Multifactor Models Do Not Explain Deviations from the CAPM," Journal of Financial Economics, 38, 3-28. 
Maddala, G. S., And W. Hu (1996): "The Pooling Problem," in Econometrics of Panel Data, ed. by L. Matyas, and P. Sevestre, pp. 307-322. Kluwer Academic Publishers.

Maddala, G. S., H. Li, And V. K. Srivastava (2001): "A Comparative Study of Different Shrinkage Estimators for Panel Data Models," Annals of Economics and Finance, $2,1-30$.

Maddala, G. S., R. P. Trost, H. Li, and F. Joutz (1997): "Estimation of Short-Run and Long-Run Elasticities of Energy Demand from Panel Data Using Shrinkage Estimators," Journal of Business and Economic Statistics, 15, 90-100.

Manski, C. (1988): "Ordinal Utility Models of Decision Making under Uncertainty," Theory and Decision, 25, 79-104.

Newey, W. K., And D. L. McFadden (1994): "Large Sample Estimation and Hypothesis Testing," in Handbook of Econometrics, Vol. 4, ed. by R. F. Engle, and D. L. McFadden. North Holland, Elsevier, Amsterdam.

Neyman, J., And E. L. Scott (1948): "Consistent Estimates Based on Partially Consistent Observations," Econometrica, 16, 1-32.

Pesaran, H., R. Smith, and K. S. Im (1996): "Dynamic Linear Models for Heterogeneous Panels," in The Econometrics of Panel Data: A Handbook of the Theory with Applications, ed. by L. Matyas, and P. Sevestre, pp. 145-195. Kluwer Academic Publishers, Dordrecht.

Pesaran, M. H. (2006): "Estimation and Inference in Large Heterogeneous Panels with a Multifactor Error Structure," Econometrica, 74, 967-1012.

Pesaran, M. H., and T. Yamagata (2008): “Testing Slope Homogeneity in Large Panels," Journal of Econometrics, 142, 50-93.

Phillips, P., And H. Moon (1999): "Linear Regression Limit Theory for Nonstationary Panel Data," Econometrica, 67, 1057-1111.

- (2000): "Nonstationary Panel Data Analysis: An Overview of Some Recent Developments," Econometric Reviews, 19, 263-286.

Phillips, P., and D. Sul (2003): "Dynamic Panel Estimation and Homogeneity Testing under Cross Section Dependence," Econometrics Journal, 6, 217-259. 
RAO, C. R. (1965): Linear Statistical Inference and Its Applications. Wiley, New York.

Rostek, M. (2010): "Quantile Maximization in Decision Theory," Review of Economic Studies, 77, 339-371.

Su, L., And Q. Chen (2013): "Testing Homogeneity in Panel Data Models with Interactive Fixed Effects," Econometric Theory, 29, 1079-1135.

Swamy, P., and G. S. Tavlas (2007): "Random Coefficient Models," in A Companion to Theoretical Econometrics, ed. by B. Baltagi, pp. 410-428. Malden: Blackwell Publishing Ltd.

Swamy, P. A. V. B. (1970): "Efficient Inference in a Random Coefficient Regression Model," Econometrica, 38, 311-323.

van Der VaArt, A. (1998): Asymptotic Statistics. Cambridge University Press, New York, New York.

Wang, H. J., And M. Fygenson (2009): "Inference for Censored Quantile Regression in Longitudinal Studies," The Annals of Statistics, 37, 756-781.

Wei, Y., And X. He (2006): "Conditional Growth Charts," The Annals of Statistics, 34, 2069-2097.

Yoon, J., And A. F. GalvaO (2014): "Robust Inference for Panel Quantile Regression Models with Individual Fixed Effects and Serial Correlation," mimeo. 


\section{Appendix}

Proof of Theorem 1.1. For the Swamy type test

$$
\begin{aligned}
& \widehat{S}= \sum_{i=1}^{n}\left(\widehat{\boldsymbol{\beta}}_{i}-\widehat{\boldsymbol{\beta}}\right)^{\top}\left(\frac{\widehat{V}_{i}}{T}\right)^{-1}\left(\widehat{\boldsymbol{\beta}}_{i}-\widehat{\boldsymbol{\beta}}\right) \\
&= \sum_{i=1}^{n}\left(\sqrt{T}\left(\widehat{\boldsymbol{\beta}}_{i}-\boldsymbol{\beta}\right)-\sqrt{T}(\widehat{\boldsymbol{\beta}}-\boldsymbol{\beta})\right)^{\top} \widehat{V}_{i}^{-1}\left(\sqrt{T}\left(\widehat{\boldsymbol{\beta}}_{i}-\boldsymbol{\beta}\right)-\sqrt{T}(\widehat{\boldsymbol{\beta}}-\boldsymbol{\beta})\right) \\
&= \sum_{i=1}^{n} \sqrt{T}\left(\widehat{\boldsymbol{\beta}}_{i}-\boldsymbol{\beta}\right)^{\top} \widehat{V}_{i}^{-1} \sqrt{T}\left(\widehat{\boldsymbol{\beta}}_{i}-\boldsymbol{\beta}\right)^{\top}-2 \sqrt{T}(\widehat{\boldsymbol{\beta}}-\boldsymbol{\beta})^{\top} \widehat{V}_{i}^{-1} \sqrt{T}\left(\widehat{\boldsymbol{\beta}}_{i}-\boldsymbol{\beta}\right)+\sqrt{T}(\widehat{\boldsymbol{\beta}}-\boldsymbol{\beta}) \widehat{V}_{i}^{-1} \sqrt{T}(\widehat{\boldsymbol{\beta}}-\boldsymbol{\beta}) \\
&= \sum_{i=1}^{n} \sqrt{T}\left(\widehat{\boldsymbol{\beta}}_{i}-\boldsymbol{\beta}\right)^{\top} \widehat{V}_{i}^{-1} \sqrt{T}\left(\widehat{\boldsymbol{\beta}}_{i}-\boldsymbol{\beta}\right)^{\top}-2\left[\left(\sum_{i=1}^{n} \widehat{V}_{i}^{-1}\right)^{-1} \sum_{i=1}^{n} \widehat{V}_{i}^{-1}\left(\widehat{\boldsymbol{\beta}}_{i}-\boldsymbol{\beta}\right)\right]^{\top} \sum_{i=1}^{n} \widehat{V}_{i}^{-1} \sqrt{T}\left(\widehat{\boldsymbol{\beta}}_{i}-\boldsymbol{\beta}\right) \\
&+\left[\left(\sum_{i=1}^{n} \widehat{V}_{i}^{-1}\right)^{-1} \sum_{i=1}^{n} \widehat{V}_{i}^{-1}\left(\widehat{\boldsymbol{\beta}}_{i}-\boldsymbol{\beta}\right)\right]^{\top} \sum_{i=1}^{n} \widehat{V}_{i}^{-1}\left[\left(\sum_{i=1}^{n} \widehat{V}_{i}^{-1}\right)^{-1} \sum_{i=1}^{n} \widehat{V}_{i}^{-1}\left(\widehat{\boldsymbol{\beta}}_{i}-\boldsymbol{\beta}\right)\right] \\
&= \sum_{i=1}^{n} \sqrt{T}\left(\widehat{\boldsymbol{\beta}}_{i}-\boldsymbol{\beta}\right)^{\top} \widehat{V}_{i}^{-1} \sqrt{T}\left(\widehat{\boldsymbol{\beta}}_{i}-\boldsymbol{\beta}\right)^{\top}-\left[\sum_{i=1}^{n} \widehat{V}_{i}^{-1}\left(\widehat{\boldsymbol{\beta}}_{i}-\boldsymbol{\beta}\right)\right]^{\top}\left(\sum_{i=1}^{n} \widehat{V}_{i}^{-1}\right)^{-1} \sum_{i=1}^{n} \widehat{V}_{i}^{-1} \sqrt{T}\left(\widehat{\boldsymbol{\beta}}_{i}-\boldsymbol{\beta}\right) \\
& \stackrel{d}{\rightarrow} \sum_{i=1}^{n} Z_{i}^{\top} V_{i}^{-1} Z_{i}-\sum_{i=1}^{n} Z_{i}^{\top} V_{i}^{-1}\left(\sum_{i=1}^{n} V_{i}^{-1}\right)^{-1} \sum_{i=1}^{n} V_{i}^{-1} Z_{i},
\end{aligned}
$$

where $Z_{i}$ are i.i.d. normal distributions with mean zero and variance $V_{i}$. The fact that $\sqrt{T}\left(\widehat{\boldsymbol{\beta}}_{i}-\boldsymbol{\beta}\right) \stackrel{d}{\rightarrow} Z_{i}$ is by the standard argument as in Koenker (2005) for i.i.d. data. Thus, the asymptotic distribution of $\widehat{S}$ is $\chi^{2}(k(n-1))$ for fixed $n$ and $T$ tending to infinity. Intuitively, the degree of freedom of the $\chi^{2}$ distribution should be $k n$ instead of $k(n-1)$. However, because we are using $\widehat{\boldsymbol{\beta}}$ for $\widehat{S}$ rather than $\boldsymbol{\beta}$, the true value of the slope parameter, $k$ degrees of freedom is lost. Therefore the degree of freedom of the $\chi^{2}$ distribution is $k(n-1)$. For more details of the Swamy type test, see pp. 149 - 153 and 323 - 324 of Rao (1965).

Proof of Theorem 1.2. Now we consider the standardized Swamy test, $\widehat{\Delta}=\sqrt{n} \frac{\frac{1}{n} \widehat{\frac{S}{n}-k}}{\sqrt{2 k}}$, for $(T, n)_{s e q} \rightarrow \infty$. To show the result, we first fix $n$. From the proof of Theorem 1.1, we know that for fixed $n, \widehat{S} \stackrel{d}{\rightarrow} \chi^{2}(k(n-1))$ as $T \rightarrow \infty$. By continuous mapping theorem, $\sqrt{n} \frac{\frac{1}{n} \widehat{S}-k}{\sqrt{2 k}} \stackrel{d}{\rightarrow} \sqrt{n} \frac{\frac{1}{n} \chi^{2}(k(n-1))-k}{\sqrt{2 k}}$ as $T$ tends to infinity.

Now we work with $\sqrt{n} \frac{\frac{1}{n} \chi^{2}(k(n-1))-k}{\sqrt{2 k}}$ and derive the asymptotic distribution as $n \rightarrow \infty$. To this end, we transform $\sqrt{n} \frac{\frac{1}{n} \chi^{2}(k(n-1))-k}{\sqrt{2 k}}$ as follows. 


$$
\begin{aligned}
\sqrt{n} \frac{\frac{1}{n} \chi^{2}(k(n-1))-k}{\sqrt{2 k}} & =\frac{\chi^{2}(k(n-1))-n k}{\sqrt{2 n k}} \\
& =\frac{\chi^{2}(k(n-1))-k(n-1)-k}{\sqrt{2(n-1) k}} \frac{\sqrt{2(n-1) k}}{\sqrt{2 n k}} \\
& =\frac{\chi^{2}(k(n-1))-k(n-1)}{\sqrt{2(n-1) k}} \frac{\sqrt{2(n-1) k}}{\sqrt{2 n k}}-\frac{k}{\sqrt{2(n-1) k}} \frac{\sqrt{2(n-1) k}}{\sqrt{2 n k}} .
\end{aligned}
$$

Using the fact that $\frac{\chi^{2}(\nu)-\nu}{\sqrt{2 \nu}} \stackrel{d}{\rightarrow} N(0,1)$ as $\nu \rightarrow \infty$, we have

$$
\sqrt{n} \frac{\frac{1}{n} \chi^{2}(k(n-1))-k}{\sqrt{2 k}} \stackrel{d}{\rightarrow} N(0,1)
$$

as $n \rightarrow \infty$. Hence, we conclude that $\widehat{\Delta} \stackrel{d}{\rightarrow} N(0,1)$ as $(T, n)_{\text {seq }} \rightarrow \infty$.

The proof of Theorem 1.3 is more involved. Denote $\mathbb{S}_{i T}(\boldsymbol{\theta}):=\frac{1}{T} \sum_{t=1}^{T} \psi_{\tau}\left(y_{i t}-\boldsymbol{X}_{i t}^{\top} \boldsymbol{\theta}\right) \boldsymbol{X}_{i t}$, where $\psi_{\tau}(u):=\tau-1\{u \leq 0\}, \boldsymbol{X}_{i t}=\left(1, \boldsymbol{x}_{i t}\right)^{\top}$, and $\boldsymbol{\theta}_{i 0}:=\left(\alpha_{i 0}, \boldsymbol{\beta}_{i 0}^{\top}\right)^{\top}$. Let $\mathbb{S}_{i}(\boldsymbol{\theta}):=$ $\mathrm{E}\left(\mathbb{S}_{i T}(\boldsymbol{\theta})\right)=\mathrm{E}\left[\tau-F_{i}\left(\boldsymbol{X}_{i 1}\left(\boldsymbol{\theta}-\boldsymbol{\theta}_{i 0}\right) \mid \boldsymbol{X}_{i 1}\right)\right]$. It is well know that the term $\sqrt{T}\left(\left[\mathbb{S}_{i T}\left(\widehat{\boldsymbol{\theta}}_{i}\right)-\right.\right.$ $\left.\left.\mathbb{S}_{i T}\left(\boldsymbol{\theta}_{i 0}\right)\right]-\left[\mathbb{S}_{i}\left(\widehat{\boldsymbol{\theta}}_{i}\right)-\mathbb{S}_{i}\left(\boldsymbol{\theta}_{i 0}\right)\right]\right)=o_{p}(1)$ for each individual since it is stochastic equicontinuous and $\widehat{\boldsymbol{\theta}}_{i} \stackrel{p}{\rightarrow} \boldsymbol{\theta}$. However, for the panel data, we need to consider the order of $\max _{1 \leq i \leq n}\left\{\left[\mathbb{S}_{i T}\left(\widehat{\boldsymbol{\theta}}_{i}\right)-\mathbb{S}_{i T}\left(\boldsymbol{\theta}_{i 0}\right)\right]-\left[\mathbb{S}_{i}\left(\widehat{\boldsymbol{\theta}}_{i}\right)-\mathbb{S}_{i}\left(\boldsymbol{\theta}_{i 0}\right)\right]\right\}$. The following lemmas provide such an order. They are auxiliary results to establish the result in Theorem 1.3. These lemmas, and corresponding proofs, are Lemmas 1, 4, and 5, respectively, in Galvao and Wang (2015). We state these results for completeness.

Lemma 6.1. Under Conditions A1-A3, we have $\max _{1 \leq i \leq n}\left\|\widehat{\boldsymbol{\theta}}_{i}-\boldsymbol{\theta}_{i 0}\right\|=o_{p}(1)$ as $(T, n) \rightarrow \infty$ and $\frac{\log n}{T} \rightarrow 0$.

Lemma 6.2. If $\max _{1 \leq i \leq n}\left\|\widehat{\boldsymbol{\theta}}_{i}-\boldsymbol{\theta}_{i 0}\right\|=O_{p}\left(\delta_{n}\right)$, where $\lim _{n \rightarrow \infty} \delta_{n}=0$, then under Conditions $A 1-A 5$, we have $\max _{1 \leq i \leq n}\left\{\left[\mathbb{S}_{i T}\left(\widehat{\boldsymbol{\theta}}_{i}\right)-\mathbb{S}_{i T}\left(\boldsymbol{\theta}_{i 0}\right)\right]-\left[\mathbb{S}_{i}\left(\widehat{\boldsymbol{\theta}}_{i}\right)-\mathbb{S}_{i}\left(\boldsymbol{\theta}_{i 0}\right)\right]\right\}=O_{p}\left(d_{n}\right)$, where $d_{n}=$ $\frac{\left|\log \delta_{n}\right|}{T} \vee \sqrt{\frac{\delta_{n}\left|\log \delta_{n}\right|}{T}}$.

Lemma 6.3. Under the conditions of Lemma 6.2, we have $\max _{1 \leq i \leq n}\left\|\widehat{\boldsymbol{\theta}}_{i}-\boldsymbol{\theta}_{i 0}\right\|=O_{p}\left(\sqrt{\frac{\log n}{T}}\right)$.

From Lemmas 6.2 and 6.3 , we notice that $d_{n}=\frac{(\log n)^{1 / 4} \sqrt{\left|\log \delta_{n}\right|}}{T^{3 / 4}}$. Now we are ready to prove Theorem 1.3. 
Proof of Theorem 1.3. Expanding $\mathbb{S}_{i}\left(\widehat{\boldsymbol{\theta}}_{i}\right)$ around $\boldsymbol{\theta}_{i 0}$ and using Lemma 2.12 of van der Vaart (1998), we obtain

$$
\mathbb{S}_{i}\left(\widehat{\boldsymbol{\theta}}_{i}\right)=\mathbb{S}_{i}\left(\boldsymbol{\theta}_{i 0}\right)+\frac{\partial \mathbb{S}_{i}\left(\boldsymbol{\theta}_{i 0}\right)}{\partial \boldsymbol{\theta}_{i}}\left(\widehat{\boldsymbol{\theta}}_{i}-\boldsymbol{\theta}_{i 0}\right)+O_{p}\left(\left(\widehat{\boldsymbol{\theta}}_{i}-\boldsymbol{\theta}_{i 0}\right)^{2}\right)
$$

After rearranging and noting that $\Gamma_{i}=\frac{\partial \mathbb{S}_{i}\left(\boldsymbol{\theta}_{i 0}\right)}{\partial \boldsymbol{\theta}_{i}}$, we have

$$
\begin{aligned}
\widehat{\boldsymbol{\theta}}_{i}-\boldsymbol{\theta}_{i 0}= & \Gamma_{i}^{-1}\left(\mathbb{S}_{i}\left(\widehat{\boldsymbol{\theta}}_{i}\right)-\mathbb{S}_{i}\left(\boldsymbol{\theta}_{i 0}\right)+O_{p}\left(\left(\widehat{\boldsymbol{\theta}}_{i}-\boldsymbol{\theta}_{i 0}\right)^{2}\right)\right) \\
= & \Gamma_{i}^{-1}\left(-\mathbb{S}_{i T}\left(\boldsymbol{\theta}_{i 0}\right)+\left(\mathbb{S}_{i}\left(\widehat{\boldsymbol{\theta}}_{i}\right)-\mathbb{S}_{i}\left(\boldsymbol{\theta}_{i 0}\right)\right)-\left(\mathbb{S}_{i T}\left(\widehat{\boldsymbol{\theta}}_{i}\right)-\mathbb{S}_{i T}\left(\boldsymbol{\theta}_{i 0}\right)\right)+\mathbb{S}_{i T}\left(\widehat{\boldsymbol{\theta}}_{i}\right)\right. \\
& \left.+O_{p}\left(\left(\widehat{\boldsymbol{\theta}}_{i}-\boldsymbol{\theta}_{i 0}\right)^{2}\right)\right) \\
= & -\Gamma_{i}^{-1} \mathbb{S}_{i T}\left(\boldsymbol{\theta}_{i 0}\right)-\Gamma_{i}^{-1}\left[\left(\mathbb{S}_{i T}\left(\widehat{\boldsymbol{\theta}}_{i}\right)-\mathbb{S}_{i T}\left(\boldsymbol{\theta}_{i 0}\right)\right)-\left(\mathbb{S}_{i}\left(\widehat{\boldsymbol{\theta}}_{i}\right)-\mathbb{S}_{i}\left(\boldsymbol{\theta}_{i 0}\right)\right)\right]+\Gamma_{i}^{-1} \mathbb{S}_{i T}\left(\widehat{\boldsymbol{\theta}}_{i}\right) \\
& +\Gamma_{i}^{-1} O_{p}\left(\left(\widehat{\boldsymbol{\theta}}_{i}-\boldsymbol{\theta}_{i 0}\right)^{2}\right) .
\end{aligned}
$$

Using equality (6.1), and Lemmas 6.2 and 6.3, we have for each $i$,

$$
\begin{aligned}
\widehat{\boldsymbol{\beta}}_{i}-\boldsymbol{\beta}_{i 0} & =-\Xi \Gamma_{i}^{-1} \mathbb{S}_{i T}\left(\boldsymbol{\theta}_{i 0}\right)+O_{p}\left(d_{n}\right)+O_{p}\left(\frac{1}{T}\right)+\Xi \Gamma_{i}^{-1} O_{p}\left(\left(\widehat{\boldsymbol{\theta}}_{i}-\boldsymbol{\theta}_{i 0}\right)^{2}\right) \\
& =-\Xi \Gamma_{i}^{-1} \mathbb{S}_{i T}\left(\boldsymbol{\theta}_{i 0}\right)+O_{p}\left(d_{n}\right)
\end{aligned}
$$

Note that the remainder term $O_{p}\left(d_{n}\right)$ depends on $n$ only. So it is a uniform version of a Bahadur representation.

Let $\widehat{V}_{i}=\Xi \widehat{\widetilde{V}}_{i} \Xi^{\top}$ and note that $\widehat{V}_{i}=V_{i}+O_{p}\left(T^{-1 / 2} h_{n}^{-1 / 2}\right)$. Now we rewrite $\frac{1}{\sqrt{n}} \widehat{S}$.

$$
\begin{aligned}
\frac{1}{\sqrt{n}} \widehat{S}= & \frac{1}{\sqrt{n}} \sum_{i=1}^{n}\left(\widehat{\boldsymbol{\beta}}_{i}-\widehat{\boldsymbol{\beta}}\right)^{\top}\left(\frac{\widehat{V}_{i}}{T}\right)^{-1}\left(\widehat{\boldsymbol{\beta}}_{i}-\widehat{\boldsymbol{\beta}}\right) \\
= & \frac{1}{\sqrt{n}} \sum_{i=1}^{n} \widehat{\boldsymbol{\beta}}_{i}^{\top}\left(\frac{\widehat{V}_{i}}{T}\right)^{-1} \widehat{\boldsymbol{\beta}}_{i}-\frac{1}{\sqrt{n}}\left(\sum_{i=1}^{n}\left(\frac{\widehat{V}_{i}}{T}\right)^{-1} \widehat{\boldsymbol{\beta}}_{i}\right)^{\top}\left(\sum_{i=1}^{n}\left(\frac{\widehat{V}_{i}}{T}\right)^{-1}\right)^{-1}\left(\sum_{i=1}^{n}\left(\frac{\widehat{V}_{i}}{T}\right)^{-1} \widehat{\boldsymbol{\beta}}_{i}\right) \\
= & \frac{1}{\sqrt{n}} \sum_{i=1}^{n}\left(\widehat{\boldsymbol{\beta}}_{i}-\boldsymbol{\beta}_{0}\right)^{\top}\left(\frac{\widehat{V}_{i}}{T}\right)^{-1}\left(\widehat{\boldsymbol{\beta}}_{i}-\boldsymbol{\beta}_{0}\right) \\
& -\frac{1}{\sqrt{n}}\left(\sum_{i=1}^{n}\left(\frac{\widehat{V}_{i}}{T}\right)^{-1}\left(\widehat{\boldsymbol{\beta}}_{i}-\boldsymbol{\beta}_{0}\right)\right)^{\top}\left(\sum_{i=1}^{n}\left(\frac{\widehat{V}_{i}}{T}\right)^{-1}\right)^{-1}\left(\sum_{i=1}^{n}\left(\frac{\widehat{V}_{i}}{T}\right)^{-1}\left(\widehat{\boldsymbol{\beta}}_{i}-\boldsymbol{\beta}_{0}\right)\right) .
\end{aligned}
$$


For the first term, we have

$$
\begin{aligned}
& \frac{1}{\sqrt{n}} \sum_{i=1}^{n}\left(\widehat{\boldsymbol{\beta}}_{i}-\boldsymbol{\beta}_{0}\right)^{\top}\left(\frac{\widehat{V}_{i}}{T}\right)^{-1}\left(\widehat{\boldsymbol{\beta}}_{i}-\boldsymbol{\beta}_{0}\right) \\
= & \frac{1}{\sqrt{n}} \sum_{i=1}^{n}\left(\Xi \Gamma_{i}^{-1} \sqrt{T} \mathbb{S}_{i T}\left(\boldsymbol{\theta}_{i 0}\right)+O_{p}\left(\sqrt{T} d_{n}\right)\right)^{\top}\left(V_{i}^{-1}+O_{p}\left(T^{-1 / 2} h_{n}^{-1 / 2}\right)\right)\left(\Xi \Gamma_{i}^{-1} \sqrt{T} \mathbb{S}_{i T}\left(\boldsymbol{\theta}_{i 0}\right)+O_{p}\left(\sqrt{T} d_{n}\right)\right) \\
= & \frac{1}{\sqrt{n}} \sum_{i=1}^{n}\left(\Xi \Gamma_{i}^{-1} \sqrt{T} \mathbb{S}_{i T}\left(\boldsymbol{\theta}_{i 0}\right)\right)^{\top} V_{i}^{-1}\left(\Xi \Gamma_{i}^{-1} \sqrt{T} \mathbb{S}_{i T}\left(\boldsymbol{\theta}_{i 0}\right)\right)+O_{p}\left(\sqrt{n T} d_{n} \vee T^{-1 / 2} h_{n}^{-1 / 2}\right) .
\end{aligned}
$$

Because $\frac{\sqrt{n}(\log n)^{1 / 4} \sqrt{\left|\log \delta_{n}\right|}}{T^{1 / 4}} \rightarrow 0$, the second term in the line above is $o_{p}(1)$.

Regarding the second term, we have

$$
\begin{aligned}
& \frac{1}{\sqrt{n}}\left(\frac{\sum_{i=1}^{n}\left(V_{i}^{-1}+O_{p}\left(T^{-1 / 2} h_{n}^{-1 / 2}\right)\right)\left(\Xi \Gamma_{i}^{-1} \sqrt{T} \mathbb{S}_{i T}\left(\boldsymbol{\theta}_{i 0}\right)+O_{p}\left(\sqrt{T} d_{n}\right)\right)}{\sqrt{n}}\right) \times \\
& \left(\frac{\sum_{i=1}^{n}\left(V_{i}^{-1}+O_{p}\left(T^{-1 / 2} h_{n}^{-1 / 2}\right)\right)}{n}\right)^{-1}\left(\frac{\sum_{i=1}^{n}\left(V_{i}^{-1}+O_{p}\left(T^{-1 / 2} h_{n}^{-1 / 2}\right)\right)\left(\Xi \Gamma_{i}^{-1} \sqrt{T} \mathbb{S}_{i T}\left(\boldsymbol{\theta}_{i 0}\right)+O_{p}\left(\sqrt{T} d_{n}\right)\right)}{\sqrt{n}}\right) \\
= & \frac{1}{\sqrt{n}}\left(\frac{\sum_{i=1}^{n} V_{i}^{-1} \Xi \Gamma_{i}^{-1} \sqrt{T} \mathbb{S}_{i T}\left(\boldsymbol{\theta}_{i 0}\right)}{\sqrt{n}}+O_{p}\left(T^{-1 / 2} h_{n}^{-1 / 2} \vee \sqrt{n T} d_{n}\right)\right)^{\top}\left(\left(\frac{1}{n} \sum_{i=1}^{n} V_{i}^{-1}\right)^{-1}+O_{p}\left(T^{-1 / 2} h_{n}^{-1 / 2}\right)\right) \\
& \times\left(\frac{\sum_{i=1}^{n} V_{i}^{-1} \Xi \Gamma_{i}^{-1} \sqrt{T} \mathbb{S}_{i T}\left(\boldsymbol{\theta}_{i 0}\right)}{\sqrt{n}}+O_{p}\left(T^{-1 / 2} h_{n}^{-1 / 2} \vee \sqrt{n T} d_{n}\right)\right) \\
= & \frac{1}{\sqrt{n}}\left(\frac{\sum_{i=1}^{n} V_{i}^{-1} \Xi \Gamma_{i}^{-1} \sqrt{T} \mathbb{S}_{i T}\left(\boldsymbol{\theta}_{i 0}\right)}{\sqrt{n}}\right)^{\top}\left(\frac{1}{n} \sum_{i=1}^{n} V_{i}^{-1}\right)^{-1}\left(\frac{\sum_{i=1}^{n} V_{i}^{-1} \Xi \Gamma_{i}^{-1} \sqrt{T} \mathbb{S}_{i T}\left(\boldsymbol{\theta}_{i 0}\right)}{\sqrt{n}}\right) \\
& +O_{p}\left(T^{-1 / 2} h_{n}^{-1 / 2} \vee \sqrt{T} d_{n}\right) \\
= & O_{p}\left(\frac{1}{\left.\sqrt{n} \vee T^{-1 / 2} h_{n}^{-1 / 2} \vee \sqrt{T} d_{n}\right) .}\right.
\end{aligned}
$$

Therefore, the second term is $o_{p}(1)$ since $\frac{\sqrt{n}(\log n)^{1 / 4} \sqrt{\left|\log \delta_{n}\right|}}{T^{1 / 4}} \rightarrow 0$.

Consequently we can write

$$
\begin{aligned}
\frac{1}{\sqrt{n}} \widehat{S} & =\frac{1}{\sqrt{n}} \sum_{i=1}^{n}\left(\Xi \Gamma_{i}^{-1} \sqrt{T} \mathbb{S}_{i T}\left(\boldsymbol{\theta}_{i 0}\right)\right)^{\top} V_{i}^{-1}\left(\Xi \Gamma_{i}^{-1} \sqrt{T} \mathbb{S}_{i T}\left(\boldsymbol{\theta}_{i 0}\right)\right)+o_{p}(1) \\
& :=\frac{1}{\sqrt{n}} \sum_{i=1}^{n} \widehat{z}_{i}+o_{p}(1) .
\end{aligned}
$$

Thus, for the standardized Swamy test, in the case when $(T, n) \rightarrow \infty$,

$$
\widehat{\Delta}:=\sqrt{n} \frac{\frac{1}{n} \widehat{S}-k}{\sqrt{2 k}}=\frac{\frac{1}{\sqrt{n}} \widehat{S}-\sqrt{n} k}{\sqrt{2 k}}=\frac{\frac{1}{\sqrt{n}} \sum_{i=1}^{n} \widehat{z}_{i}+o_{p}(1)-\sqrt{n} k}{\sqrt{2 k}}=\frac{1}{\sqrt{n}} \sum_{i=1}^{n}\left(\frac{\widehat{z}_{i}-k}{\sqrt{2 k}}\right)+o_{p}(1) .
$$


For some small positive constant $\bar{\epsilon}$, we have $\mathrm{E}\left|\widehat{z}_{i}\right|^{2+\bar{\epsilon}}<\bar{K}<\infty$ by condition A2. Also, $\mathrm{E} \widehat{z}_{i}=k$ for all $i$. Therefore, by Lindberg-Feller Central Limit Theorem, we have

$$
\widehat{\Delta} \stackrel{d}{\rightarrow} N\left(0, \widehat{g}^{2}\right)
$$

where $\widehat{g}^{2}:=\lim _{n \rightarrow \infty} \frac{1}{n} \sum_{i=1}^{n}\left(\frac{\operatorname{Var}\left(\widehat{z}_{i}\right)}{2 k}\right)$. Finally, it just remains to show that $\widehat{g}^{2}=1$, and the proof is complete.

Since $\operatorname{Var}\left(\widehat{z}_{i}\right)=\mathrm{E}\left(\widehat{z}_{i}\right)^{2}-k^{2}$, we need to verify that $\mathrm{E}\left(\widehat{z}_{i}\right)^{2}=2 k+k^{2}+o(1)$ uniformly across $i$ as $T \rightarrow \infty$. Let $\boldsymbol{Z}_{i t}=V_{i}^{-1 / 2} \Xi \Gamma_{i}^{-1} \psi_{\tau}\left(y_{i t}-\boldsymbol{X}_{i t}^{\top} \boldsymbol{\theta}_{i 0}\right) \boldsymbol{X}_{i t}$, we have $\mathrm{E} \boldsymbol{Z}_{i t}=\mathbf{0}$, and $\mathrm{E} \boldsymbol{Z}_{i t} \boldsymbol{Z}_{i t}^{\top}=\boldsymbol{I}$. Let $Z_{i t l}$ be the $l$ th element of $\boldsymbol{Z}_{i t}$. Since $Z_{i t l}$ is linear combination of the vector $\boldsymbol{X}_{i t}$, and $\psi_{\tau}(\cdot)$ is a uniformly bounded function, it follows that $\mathrm{E} Z_{i t l}^{2} Z_{i t m}^{2}$ and $\mathrm{E} Z_{i t l}^{4}$ are both uniformly bounded for any $l$ and $m$. Note that we do not have that $\boldsymbol{Z}_{i t}$ follows normal distribution. Consequently, the elements in each vector $\boldsymbol{Z}_{i t}$ need not be independent, although they are uncorrelated. Nonetheless, $\boldsymbol{Z}_{i t}$ are independent across $i$ and $t$ by Condition A1. Now we compute $\mathrm{E}\left(\widehat{z}_{i}\right)^{2}$.

$$
\begin{aligned}
& \mathrm{E}\left(\widehat{z}_{i}\right)^{2}=T^{2} \mathrm{E}\left[\mathbb{S}_{i T}^{\top}\left(\boldsymbol{\theta}_{i 0}\right) \Gamma_{i}^{-1} \Xi^{\top} V_{i}^{-1} \Xi \Gamma_{i}^{-1} \mathbb{S}_{i T}\left(\boldsymbol{\theta}_{i 0}\right)\right]^{2} \\
= & \frac{1}{T^{2}} \mathrm{E}\left[\sum_{t=1}^{T} \psi_{\tau}\left(y_{i t}-\boldsymbol{X}_{i t}^{\top} \boldsymbol{\theta}_{i 0}\right) \boldsymbol{X}_{i t}^{\top} \Gamma_{i}^{-1} \Xi^{\top} V_{i}^{-1 / 2} \sum_{t=1}^{T} V_{i}^{-1 / 2} \Xi \Gamma_{i}^{-1} \psi_{\tau}\left(y_{i t}-\boldsymbol{X}_{i t}^{\top} \boldsymbol{\theta}_{i 0}\right) \boldsymbol{X}_{i t}\right]^{2} \\
= & \frac{1}{T^{2}} \mathrm{E}\left[\left(\sum_{t=1}^{T} \boldsymbol{Z}_{i t}\right)^{\top} \sum_{t=1}^{T} \boldsymbol{Z}_{i t}\right]^{2}=\frac{1}{T^{2}} \mathrm{E}\left[\sum_{l=1}^{k}\left(\sum_{t=1}^{T} Z_{i t l}\right)^{2}\right]^{2} \\
= & \frac{1}{T^{2}} \sum_{l=1}^{k} \mathrm{E}\left(\sum_{t=1}^{T} Z_{i t l}\right)^{4}+\frac{2}{T^{2}} \sum_{m<l} \mathrm{E}\left(\sum_{t=1}^{T} Z_{i t l}\right)^{2}\left(\sum_{t=1}^{T} Z_{i t m}\right)^{2} \\
= & \frac{1}{T^{2}} \sum_{l=1}^{k}\left(\sum_{t=1}^{T} \mathrm{E} Z_{i t l}^{4}+3 T(T-1) \mathrm{E} Z_{i 1 l}^{2} \mathrm{E} Z_{i 1 l}^{2}\right) \\
& +\frac{2}{T^{2}} \sum_{m<l} \mathrm{E}\left(\sum_{t=1}^{T} Z_{i t l}^{2}+2 \sum_{s<t} Z_{i t l} Z_{i s l}\right)\left(\sum_{t=1}^{T} Z_{i t m}^{2}+2 \sum_{s<t} Z_{i t m} Z_{i s m}\right) \\
= & 3 k+\frac{1}{T} \sum_{l=1}^{k}\left(\mathrm{E} Z_{i t l}^{4}-3\right)+\frac{2}{T^{2}} \sum_{m<l} \mathrm{E}\left(\sum_{t=1}^{T} Z_{i t l}^{2} \sum_{t=1}^{T} Z_{i t m}^{2}+2 \sum_{s<t} Z_{i t l} Z_{i s l} \sum_{t=1}^{T} Z_{i t m}^{2}\right. \\
& \left.+2 \sum_{s<t} Z_{i t m} Z_{i s m} \sum_{t=1}^{T} Z_{i t l}^{2}+4 \sum_{s<t} Z_{i t l} Z_{i s l} \sum_{s<t} Z_{i t m} Z_{i s m}\right)
\end{aligned}
$$

Line (6.2) follows since the expectations of all other terms from the expansion of the fourth 
order polynomial are zeros. And the third term equals

$$
\begin{aligned}
& \frac{2}{T^{2}} \sum_{m<l} \mathrm{E}\left(\sum_{t=1}^{T} \sum_{s=1}^{T} Z_{i t l}^{2} Z_{i s m}^{2}+2 \sum_{s<t} \sum_{r=1}^{T} Z_{i t l} Z_{i s l} Z_{i r m}^{2}+2 \sum_{s<t} \sum_{r=1}^{T} Z_{i t m} Z_{i s m} Z_{i r l}^{2}+4 \sum_{s<t} \sum_{r<q} Z_{i t l} Z_{i s l} Z_{i r m} Z_{i q m}\right) \\
= & \frac{2}{T^{2}} \sum_{m<l}\left(\sum_{t=1}^{T} \mathrm{E} Z_{i t l}^{2} Z_{i t m}^{2}+T(T-1)+4 \sum_{s<t} \mathrm{E} Z_{i t l} Z_{i t m} Z_{i s l} Z_{i s m}\right) \\
= & k(k-1)+\frac{2}{T^{2}} \sum_{m<l}\left(\sum_{t=1}^{T} \mathrm{E} Z_{i t l}^{2} Z_{i t m}^{2}-T\right) \\
= & k(k-1)+O(1 / T) .
\end{aligned}
$$

Equality (6.3) follows because the second and the third terms in the parentheses in the line above are zeros. Equality (6.4) follows since $\mathrm{E} Z_{i t l} Z_{i t m} Z_{i s l} Z_{i s m}=\mathrm{E} Z_{i t l} Z_{i t m} \mathrm{E} Z_{i s l} Z_{i s m}=0$. Thus, $\mathrm{E}\left(\widehat{z}_{i}\right)^{2}=3 k+k(k-1)+O(1 / T)=2 k+k^{2}+O(1 / T)$.

Proof of Theorem 2.1. The proof is analogue to that of Theorem 1.1. The only difference is how to establish $\sqrt{T}\left(\widehat{\boldsymbol{\beta}}_{i}-\boldsymbol{\beta}\right) \stackrel{d}{\rightarrow} Z_{i}$. Indeed, instead of using the standard results for i.i.d. data, we apply asymptotic results for stationary $\beta$-mixing data.

Proof of Theorem 2.2. The proof uses the proof of Theorem 2.1 and follows from the same argument as that of the proof of Theorem 1.2.

Next, to prove Theorem 2.3, we state lemmas collecting intermediate results for the dependent case. These lemmas, and corresponding proofs, are Lemmas 6, 7, and 8, respectively in Galvao and Wang (2015). We state these results for completeness.

Lemma 6.4. Under Conditions A2-A3 and B1, we have $\max _{1 \leq i \leq n}\left\|\widehat{\boldsymbol{\theta}}_{i}-\boldsymbol{\theta}_{i 0}\right\|=o_{p}(1)$ as $(n, T) \rightarrow \infty$ and $\frac{\log n}{T} \rightarrow 0$.

Lemma 6.5. Assume conditions $A \mathscr{2}-A 5$ and $B 1-B 2$ hold. For any $c \in(0,1)$ and $\delta_{N}$ such that $\left|\log \delta_{N}\right| \asymp \log N$, we have we have $\max _{1 \leq i \leq n}\left\{\left[\mathbb{S}_{i T}\left(\widehat{\boldsymbol{\theta}}_{i}\right)-\mathbb{S}_{i T}\left(\boldsymbol{\theta}_{i 0}\right)\right]-\left[S_{i}\left(\widehat{\boldsymbol{\theta}}_{i}\right)-S_{i}\left(\boldsymbol{\theta}_{i 0}\right)\right]\right\}=$ $O_{p}\left(T^{-(1-c)}(\log n) \vee T^{-1 / 2} \delta_{n}^{1 / 2}(\log n)^{1 / 2}\right)$.

Lemma 6.6. Assume conditions A2 - A5 and B1-B2 hold. We have $\max _{1 \leq i \leq n}\left\|\widehat{\boldsymbol{\theta}}_{i}-\boldsymbol{\theta}_{i 0}\right\|=$ $O_{p}\left(\sqrt{\frac{\log n}{T}}\right)$ for $\beta$-mixing data.

Proof of Theorem 2.3. The proof is similar to that of Theorem 1.3. Instead of applying Lemmas 6.1, 6.2, and 6.3, we apply Lemmas 6.4, 6.5, and 6.6. 
Table 1: Empirical size and power for static location model $(\gamma=0)$ with $N(0,1)$ innovations.

\begin{tabular}{|c|c|c|c|c|c|c|c|c|c|}
\hline \multirow{3}{*}{\multicolumn{2}{|c|}{$\begin{array}{l}n \\
T\end{array}$}} & \multicolumn{4}{|c|}{100} & \multicolumn{4}{|c|}{200} \\
\hline & & \multicolumn{2}{|c|}{50} & \multicolumn{2}{|c|}{100} & \multicolumn{2}{|c|}{50} & \multicolumn{2}{|c|}{100} \\
\hline & & $S$ & $\Delta$ & $S$ & $\Delta$ & $S$ & $\Delta$ & $S$ & $\Delta$ \\
\hline$\tau$ & Bandwidth & \multicolumn{8}{|c|}{ Empirical size } \\
\hline 0.10 & $\mathrm{~B}$ & 0.033 & 0.033 & 0.049 & 0.049 & 0.069 & 0.069 & 0.054 & 0.054 \\
\hline 0.10 & HS & 0.022 & 0.022 & 0.058 & 0.058 & 0.015 & 0.015 & 0.076 & 0.076 \\
\hline 0.25 & $\mathrm{~B}$ & 0.030 & 0.029 & 0.054 & 0.054 & 0.026 & 0.026 & 0.081 & 0.081 \\
\hline 0.25 & HS & 0.024 & 0.024 & 0.036 & 0.036 & 0.014 & 0.015 & 0.033 & 0.033 \\
\hline 0.50 & B & 0.075 & 0.073 & 0.062 & 0.062 & 0.091 & 0.091 & 0.058 & 0.058 \\
\hline 0.50 & HS & 0.018 & 0.018 & 0.054 & 0.053 & 0.011 & 0.011 & 0.052 & 0.052 \\
\hline 0.75 & B & 0.030 & 0.029 & 0.029 & 0.029 & 0.021 & 0.021 & 0.078 & 0.078 \\
\hline 0.75 & HS & 0.026 & 0.026 & 0.049 & 0.048 & 0.014 & 0.014 & 0.028 & 0.028 \\
\hline 0.90 & B & 0.070 & 0.070 & 0.068 & 0.067 & 0.078 & 0.078 & 0.046 & 0.047 \\
\hline 0.90 & HS & 0.027 & 0.027 & 0.054 & 0.053 & 0.020 & 0.020 & 0.046 & 0.047 \\
\hline$\tau$ & Bandwidth & \multicolumn{8}{|c|}{ Empirical power } \\
\hline 0.10 & $\mathrm{~B}$ & 1.000 & 1.000 & 1.000 & 1.000 & 1.000 & 1.000 & 1.000 & 1.000 \\
\hline 0.10 & HS & 1.000 & 1.000 & 1.000 & 1.000 & 1.000 & 1.000 & 1.000 & 1.000 \\
\hline 0.25 & B & 1.000 & 1.000 & 1.000 & 1.000 & 1.000 & 1.000 & 1.000 & 1.000 \\
\hline 0.25 & HS & 1.000 & 1.000 & 1.000 & 1.000 & 1.000 & 1.000 & 1.000 & 1.000 \\
\hline 0.50 & B & 1.000 & 1.000 & 1.000 & 1.000 & 1.000 & 1.000 & 1.000 & 1.000 \\
\hline 0.50 & HS & 1.000 & 1.000 & 1.000 & 1.000 & 1.000 & 1.000 & 1.000 & 1.000 \\
\hline 0.75 & B & 1.000 & 1.000 & 1.000 & 1.000 & 1.000 & 1.000 & 1.000 & 1.000 \\
\hline 0.75 & HS & 1.000 & 1.000 & 1.000 & 1.000 & 1.000 & 1.000 & 1.000 & 1.000 \\
\hline 0.90 & $\mathrm{~B}$ & 1.000 & 1.000 & 1.000 & 1.000 & 1.000 & 1.000 & 1.000 & 1.000 \\
\hline 0.90 & HS & 1.000 & 1.000 & 1.000 & 1.000 & 1.000 & 1.000 & 1.000 & 1.000 \\
\hline
\end{tabular}

Notes: Monte Carlo simulations based on 1,000 replications. Bandwidth: B: Bofinger, HS: Hall-Sheather. 
Table 2: Empirical size and power for static location-scale model $(\gamma=0.1)$ with $N(0,1)$.

\begin{tabular}{|c|c|c|c|c|c|c|c|c|c|}
\hline \multirow{3}{*}{\multicolumn{2}{|c|}{$\frac{n}{T}$}} & \multicolumn{4}{|c|}{100} & \multicolumn{4}{|c|}{200} \\
\hline & & \multicolumn{2}{|c|}{50} & \multicolumn{2}{|c|}{100} & \multicolumn{2}{|c|}{50} & \multicolumn{2}{|c|}{100} \\
\hline & & $S$ & $\Delta$ & $S$ & $\Delta$ & $S$ & $\Delta$ & $S$ & $\Delta$ \\
\hline$\tau$ & Bandwidth & \multicolumn{8}{|c|}{ Empirical size } \\
\hline 0.10 & B & 0.029 & 0.029 & 0.051 & 0.051 & 0.035 & 0.035 & 0.053 & 0.053 \\
\hline 0.10 & HS & 0.037 & 0.037 & 0.050 & 0.049 & 0.073 & 0.073 & 0.057 & 0.057 \\
\hline 0.25 & $\mathrm{~B}$ & 0.029 & 0.029 & 0.071 & 0.071 & 0.028 & 0.028 & 0.056 & 0.056 \\
\hline 0.25 & HS & 0.041 & 0.041 & 0.053 & 0.053 & 0.019 & 0.019 & 0.044 & 0.044 \\
\hline 0.50 & B & 0.070 & 0.070 & 0.030 & 0.030 & 0.052 & 0.052 & 0.026 & 0.026 \\
\hline 0.50 & HS & 0.040 & 0.040 & 0.046 & 0.046 & 0.036 & 0.036 & 0.067 & 0.067 \\
\hline 0.75 & B & 0.024 & 0.024 & 0.062 & 0.062 & 0.025 & 0.025 & 0.048 & 0.048 \\
\hline 0.75 & HS & 0.029 & 0.029 & 0.059 & 0.058 & 0.033 & 0.033 & 0.060 & 0.061 \\
\hline 0.90 & B & 0.043 & 0.043 & 0.058 & 0.057 & 0.026 & 0.026 & 0.040 & 0.040 \\
\hline 0.90 & HS & 0.039 & 0.039 & 0.051 & 0.051 & 0.082 & 0.082 & 0.060 & 0.060 \\
\hline$\tau$ & Bandwidth & \multicolumn{8}{|c|}{ Empirical power } \\
\hline 0.10 & $\mathrm{~B}$ & 1.000 & 1.000 & 1.000 & 1.000 & 1.000 & 1.000 & 1.000 & 1.000 \\
\hline 0.10 & HS & 1.000 & 1.000 & 1.000 & 1.000 & 1.000 & 1.000 & 1.000 & 1.000 \\
\hline 0.25 & B & 1.000 & 1.000 & 1.000 & 1.000 & 1.000 & 1.000 & 1.000 & 1.000 \\
\hline 0.25 & HS & 1.000 & 1.000 & 1.000 & 1.000 & 1.000 & 1.000 & 1.000 & 1.000 \\
\hline 0.50 & B & 1.000 & 1.000 & 1.000 & 1.000 & 1.000 & 1.000 & 1.000 & 1.000 \\
\hline 0.50 & HS & 1.000 & 1.000 & 1.000 & 1.000 & 1.000 & 1.000 & 1.000 & 1.000 \\
\hline 0.75 & $\mathrm{~B}$ & 1.000 & 1.000 & 1.000 & 1.000 & 1.000 & 1.000 & 1.000 & 1.000 \\
\hline 0.75 & HS & 1.000 & 1.000 & 1.000 & 1.000 & 1.000 & 1.000 & 1.000 & 1.000 \\
\hline 0.90 & $\mathrm{~B}$ & 1.000 & 1.000 & 1.000 & 1.000 & 1.000 & 1.000 & 1.000 & 1.000 \\
\hline 0.90 & HS & 1.000 & 1.000 & 1.000 & 1.000 & 1.000 & 1.000 & 1.000 & 1.000 \\
\hline
\end{tabular}

Notes: Monte Carlo simulations based on 1,000 replications. Bandwidth: B: Bofinger, HS: Hall-Sheather. 
Table 3: Empirical size and power for dynamic model with $N(0,1)$ innovations.

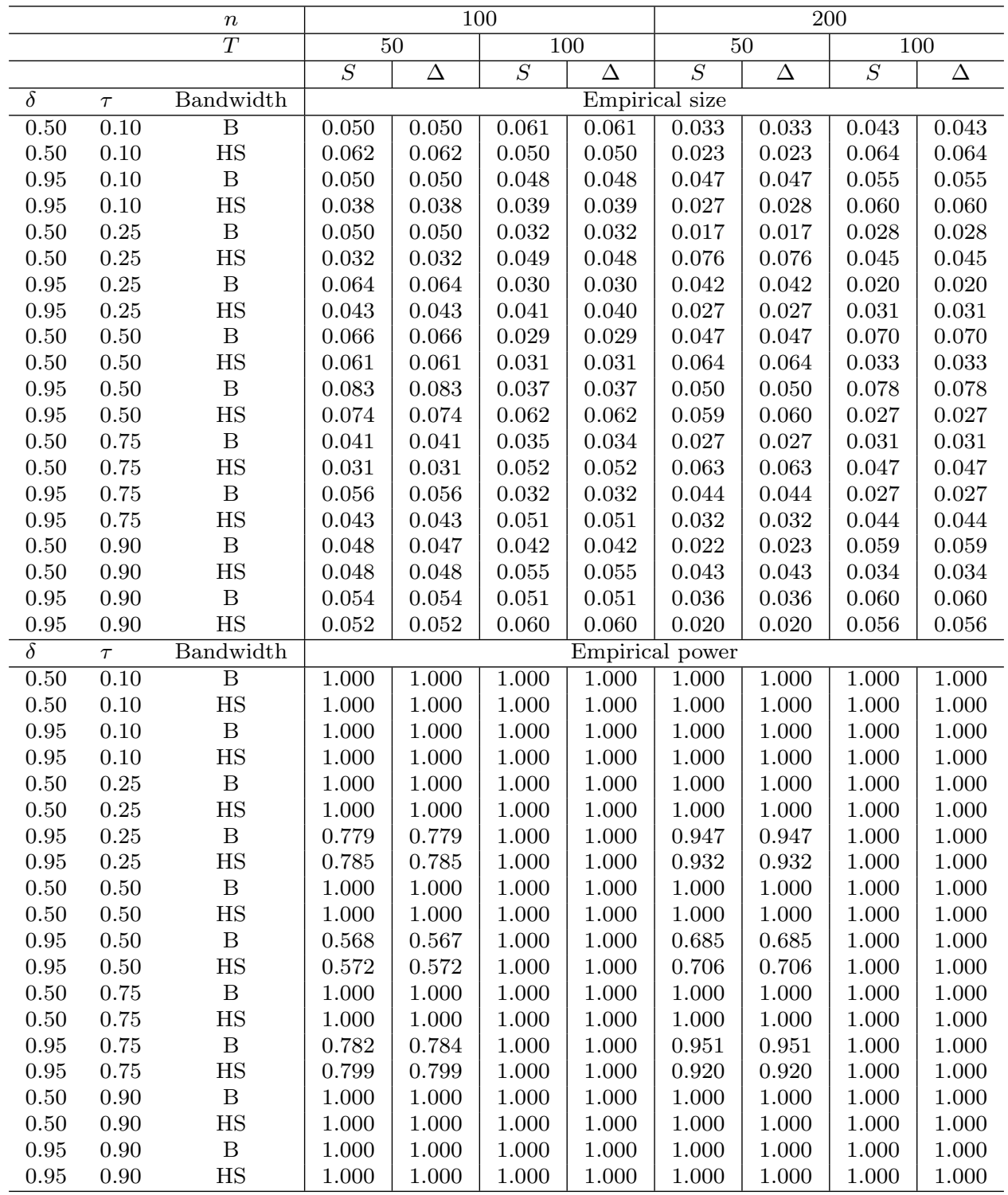

Notes: Monte Carlo simulations based on 1,000 replications. Bandwidth: B: Bofinger, HS: Hall-Sheather. 
Table 4: Summary statistics. Firm characteristics.

\begin{tabular}{cccccc}
\hline Variable & Obs & Mean & Std. Dev. & Min & Max \\
\hline RTN & 12177 & 0.003 & 0.795 & -10.17 & 10.48 \\
MDR & 12177 & 0.270 & 0.207 & 0.000 & 0.998 \\
EBITTA & 12177 & 0.112 & 0.096 & -1.466 & 1.103 \\
MB & 12177 & 1.224 & 0.925 & 0.025 & 36.91 \\
DEPTA & 12177 & 0.043 & 0.020 & 0.000 & 0.338 \\
LNTA & 12177 & 20.59 & 1.934 & 14.36 & 26.25 \\
FATA & 12177 & 0.343 & 0.188 & 0.000 & 0.935 \\
MKT & 12177 & 6.626 & 16.797 & -51.234 & 30.160 \\
\hline
\end{tabular}

Notes: Sample consists of 1970 to 2011 for 294 firms. $M D R$ : market debt ratio = book value of (short-term plus long-term) debt (Compustat items [9]+[34])/market value of assets (Compustat items $[9]+[34]+[199]^{*}[25]$ ). EBITTA: profitability: earnings before interest and taxes (Compustat items $[18]+[15]+[16]$ ), as a proportion of total assets (Compustat item [6]). $M B$ : market to book ratio of assets: book liabilities plus market value of equity (Compustat items $[9]+[34]+[10]+[199]^{*}[25]$ ) divided by book value of total assets (Compustat item [6]). DEPTA: depreciation (Compustat item [14]) as a proportion of total assets (Compustat item [6]). LNT A: log of asset size, measured in 1983 dollars (Compustat item (6)*1,000,000, deflated by the consumer price index. FATA: fixed asset proportion: property, plant, and equipment (Compustat item [8)]/total assets (Compustat Item [6]). MKT: market portfolio excess return. 
Table 5: P-values test results. Three-variable specification.

\begin{tabular}{c|ccccccccc}
\hline & \multicolumn{1}{c}{ joint } & \multicolumn{1}{c}{ MB } & \multicolumn{2}{c}{ LNTA } & \multicolumn{2}{c}{ MKT } \\
\hline & $S$ & $\Delta$ & $S$ & $\Delta$ & $S$ & $\Delta$ & $S$ & $\Delta$ \\
\hline \multicolumn{19}{c}{ Mean based model } \\
\hline & 1.000 & 1.000 & 0.001 & 0.000 & 1.000 & 1.000 & 1.000 & 1.000 \\
\hline$\tau$ & \multicolumn{9}{c}{ Quantile regression models } \\
\hline 0.05 & 0.000 & 0.000 & 0.000 & 0.000 & 0.000 & 0.000 & 0.000 & 0.000 \\
0.10 & 0.000 & 0.000 & 0.000 & 0.000 & 0.000 & 0.000 & 1.000 & 0.999 \\
0.15 & 0.749 & 0.752 & 0.002 & 0.001 & 0.199 & 0.201 & 1.000 & 1.000 \\
0.20 & 1.000 & 1.000 & 0.670 & 0.677 & 0.999 & 0.997 & 1.000 & 1.000 \\
0.25 & 1.000 & 1.000 & 0.775 & 0.778 & 1.000 & 1.000 & 1.000 & 1.000 \\
0.30 & 1.000 & 1.000 & 0.987 & 0.983 & 1.000 & 1.000 & 1.000 & 1.000 \\
0.35 & 1.000 & 1.000 & 0.995 & 0.992 & 1.000 & 1.000 & 1.000 & 1.000 \\
0.40 & 1.000 & 1.000 & 0.998 & 0.996 & 1.000 & 1.000 & 1.000 & 1.000 \\
0.45 & 1.000 & 1.000 & 0.998 & 0.996 & 1.000 & 1.000 & 1.000 & 1.000 \\
0.50 & 1.000 & 1.000 & 0.995 & 0.993 & 1.000 & 1.000 & 1.000 & 1.000 \\
0.55 & 1.000 & 1.000 & 0.950 & 0.945 & 1.000 & 1.000 & 1.000 & 1.000 \\
0.60 & 1.000 & 1.000 & 0.851 & 0.850 & 1.000 & 1.000 & 1.000 & 1.000 \\
0.65 & 1.000 & 1.000 & 0.702 & 0.708 & 1.000 & 1.000 & 1.000 & 1.000 \\
0.70 & 1.000 & 1.000 & 0.127 & 0.125 & 1.000 & 1.000 & 1.000 & 1.000 \\
0.75 & 1.000 & 0.999 & 0.010 & 0.007 & 1.000 & 1.000 & 0.999 & 0.998 \\
0.80 & 0.125 & 0.124 & 0.000 & 0.000 & 0.822 & 0.822 & 0.237 & 0.241 \\
0.85 & 0.000 & 0.000 & 0.000 & 0.000 & 0.000 & 0.000 & 0.000 & 0.000 \\
0.90 & 0.000 & 0.000 & 0.000 & 0.000 & 0.000 & 0.000 & 0.000 & 0.000 \\
0.95 & 0.000 & 0.000 & 0.000 & 0.000 & 0.000 & 0.000 & 0.000 & 0.000 \\
\hline
\end{tabular}

Notes: See notes in Table 4. Firm-characteristics three-variable specification. Mean and quantile slope homogeneity tests across firms $i$ of the model $R T N_{i, t}=\alpha_{i}+\beta_{i, M B} M B_{i, t}+$ $\beta_{i, L N T A} L N T A_{i, t}+\beta_{i, M K T} M K T_{t}+\varepsilon_{i, t+1} . S$ is the Swamy-type test and $\Delta$ the standardized Swamy test. P-values are reported. 
Table 6: P-values test results. Three-variable specification. Subsample 1.

\begin{tabular}{c|ccccccccc}
\hline & \multicolumn{1}{c}{ joint } & \multicolumn{1}{c}{ MB } & \multicolumn{2}{c}{ LNTA } & \multicolumn{2}{c}{ MKT } \\
\hline & $S$ & $\Delta$ & $S$ & $\Delta$ & $S$ & $\Delta$ & $S$ & $\Delta$ \\
\hline \multicolumn{1}{c}{ Mean based model } \\
\hline & 1.000 & 1.000 & 0.573 & 0.588 & 1.000 & 0.999 & 1.000 & 0.999 \\
\hline$\tau$ & \multicolumn{9}{c}{ Quantile regression models } \\
\hline 0.05 & 0.000 & 0.000 & 0.000 & 0.000 & 0.000 & 0.000 & 0.001 & 0.000 \\
0.10 & 0.000 & 0.000 & 0.000 & 0.000 & 0.000 & 0.000 & 0.930 & 0.923 \\
0.15 & 0.005 & 0.004 & 0.001 & 0.000 & 0.003 & 0.001 & 0.997 & 0.993 \\
0.20 & 0.988 & 0.984 & 0.240 & 0.247 & 0.695 & 0.705 & 1.000 & 1.000 \\
0.25 & 1.000 & 1.000 & 0.219 & 0.224 & 0.996 & 0.992 & 1.000 & 1.000 \\
0.30 & 1.000 & 1.000 & 0.615 & 0.628 & 1.000 & 1.000 & 1.000 & 1.000 \\
0.35 & 1.000 & 1.000 & 0.601 & 0.615 & 1.000 & 1.000 & 1.000 & 1.000 \\
0.40 & 1.000 & 1.000 & 0.672 & 0.683 & 1.000 & 1.000 & 1.000 & 1.000 \\
0.45 & 1.000 & 1.000 & 0.741 & 0.748 & 1.000 & 1.000 & 1.000 & 1.000 \\
0.50 & 1.000 & 1.000 & 0.695 & 0.704 & 1.000 & 1.000 & 1.000 & 1.000 \\
0.55 & 1.000 & 1.000 & 0.507 & 0.522 & 1.000 & 1.000 & 1.000 & 1.000 \\
0.60 & 1.000 & 1.000 & 0.287 & 0.296 & 1.000 & 1.000 & 1.000 & 1.000 \\
0.65 & 1.000 & 1.000 & 0.274 & 0.283 & 1.000 & 1.000 & 1.000 & 1.000 \\
0.70 & 1.000 & 1.000 & 0.035 & 0.029 & 1.000 & 1.000 & 1.000 & 1.000 \\
0.75 & 0.957 & 0.952 & 0.004 & 0.002 & 0.992 & 0.987 & 0.987 & 0.981 \\
0.80 & 0.212 & 0.215 & 0.000 & 0.000 & 0.905 & 0.900 & 0.486 & 0.501 \\
0.85 & 0.000 & 0.000 & 0.000 & 0.000 & 0.000 & 0.000 & 0.004 & 0.002 \\
0.90 & 0.000 & 0.000 & 0.000 & 0.000 & 0.000 & 0.000 & 0.000 & 0.000 \\
0.95 & 0.000 & 0.000 & 0.000 & 0.000 & 0.000 & 0.000 & 0.000 & 0.000 \\
\hline
\end{tabular}

Notes: See notes in Table 4. Firm-characteristics three-variable specification. Mean and quantile slope homogeneity tests across firms $i$ of the model $R T N_{i, t+1}=\alpha_{i}+\beta_{i, M B} M B_{i, t}+$ $\beta_{i, L N T A} L N T A_{i, t}+\beta_{i, M K T} M K T_{t}+\varepsilon_{i, t+1}, i=1,2, \ldots, 294 . S$ is the Swamy-type test and $\Delta$ the standardized Swamy test. P-values are reported. Subsample of firms with average $M B$ below the median. 
Table 7: P-values test results. Three-variable specification. Subsample 2.

\begin{tabular}{c|ccccccccc}
\hline & \multicolumn{1}{c}{ joint } & \multicolumn{1}{c}{ MB } & \multicolumn{2}{c}{ LNTA } & \multicolumn{2}{c}{ MKT } \\
\hline & $S$ & $\Delta$ & $S$ & $\Delta$ & $S$ & $\Delta$ & $S$ & $\Delta$ \\
\hline \multicolumn{1}{c}{ Mean based model } \\
\hline & 0.998 & 0.997 & 0.000 & 0.000 & 1.000 & 0.999 & 1.000 & 1.000 \\
\hline$\tau$ & \multicolumn{9}{c}{ Quantile regression models } \\
\hline 0.05 & 0.000 & 0.000 & 0.000 & 0.000 & 0.000 & 0.000 & 0.000 & 0.000 \\
0.10 & 0.510 & 0.519 & 0.030 & 0.024 & 0.195 & 0.198 & 1.000 & 0.999 \\
0.15 & 1.000 & 1.000 & 0.483 & 0.499 & 0.992 & 0.987 & 1.000 & 1.000 \\
0.20 & 1.000 & 1.000 & 0.971 & 0.963 & 1.000 & 1.000 & 1.000 & 1.000 \\
0.25 & 1.000 & 1.000 & 0.996 & 0.992 & 1.000 & 1.000 & 1.000 & 1.000 \\
0.30 & 1.000 & 1.000 & 1.000 & 0.999 & 1.000 & 1.000 & 1.000 & 1.000 \\
0.35 & 1.000 & 1.000 & 1.000 & 1.000 & 1.000 & 1.000 & 1.000 & 1.000 \\
0.40 & 1.000 & 1.000 & 1.000 & 1.000 & 1.000 & 1.000 & 1.000 & 1.000 \\
0.45 & 1.000 & 1.000 & 1.000 & 1.000 & 1.000 & 1.000 & 1.000 & 1.000 \\
0.50 & 1.000 & 1.000 & 1.000 & 0.999 & 1.000 & 1.000 & 1.000 & 1.000 \\
0.55 & 1.000 & 1.000 & 0.995 & 0.991 & 1.000 & 1.000 & 1.000 & 1.000 \\
0.60 & 1.000 & 1.000 & 0.990 & 0.984 & 1.000 & 1.000 & 1.000 & 1.000 \\
0.65 & 1.000 & 1.000 & 0.945 & 0.937 & 1.000 & 1.000 & 1.000 & 1.000 \\
0.70 & 1.000 & 1.000 & 0.662 & 0.674 & 1.000 & 1.000 & 1.000 & 0.999 \\
0.75 & 0.999 & 0.999 & 0.326 & 0.338 & 0.999 & 0.996 & 0.987 & 0.981 \\
0.80 & 0.199 & 0.200 & 0.052 & 0.045 & 0.479 & 0.495 & 0.145 & 0.145 \\
0.85 & 0.000 & 0.000 & 0.000 & 0.000 & 0.000 & 0.000 & 0.000 & 0.000 \\
0.90 & 0.000 & 0.000 & 0.000 & 0.000 & 0.000 & 0.000 & 0.000 & 0.000 \\
0.95 & 0.000 & 0.000 & 0.000 & 0.000 & 0.000 & 0.000 & 0.000 & 0.000 \\
\hline
\end{tabular}

Notes: See notes in Table 4. Firm-characteristics three-variable specification. Mean and quantile slope homogeneity tests across firms $i$ of the model $R T N_{i, t+1}=\alpha_{i}+\beta_{i, M B} M B_{i, t}+$ $\beta_{i, L N T A} L N T A_{i, t}+\beta_{i, M K T} M K T_{t}+\varepsilon_{i, t+1}, i=1,2, \ldots, 294 . S$ is the Swamy-type test and $\Delta$ the standardized Swamy test. P-values are reported. Subsample of firms with average $M B$ above the median. 
Table 8: FE and FE QR estimation results. Three-variable specification.

\begin{tabular}{c|cccccc} 
variables & Mean & $\tau=0.10$ & $\tau=0.25$ & $\tau=0.50$ & $\tau=0.75$ & $\tau=0.90$ \\
\hline MB & $-0.137^{* * *}$ & $-0.194^{* * *}$ & $-0.161^{* * *}$ & $-0.140^{* * *}$ & $-0.129^{* * *}$ & $-0.116^{* * *}$ \\
& $(0.010)$ & $(0.013)$ & $(0.003)$ & $(0.000)$ & $(0.006)$ & $(0.028)$ \\
LNTA & $0.076^{* * *}$ & $0.136^{* * *}$ & $0.0886^{* * *}$ & $0.0680^{* * *}$ & $0.0415^{* * *}$ & 0.017 \\
& $(0.010)$ & $(0.014)$ & $(0.004)$ & $(0.001)$ & $(0.005)$ & $(0.018)$ \\
MKT & $0.001^{*}$ & $0.003^{* * *}$ & $0.002^{* * *}$ & $0.0001^{* * *}$ & $-0.0005^{* * *}$ & $-0.002^{* * *}$ \\
& $(0.0004)$ & $(0.0007)$ & $(0.0002)$ & $(0.000)$ & $(0.0002)$ & $(0.0007)$ \\
\hline
\end{tabular}

Notes: See notes in Table 4. Firm-characteristics three-variable specification. Mean and quantile FE model $R T N_{i, t+1}=\alpha_{i}+\beta_{M B} M B_{i, t}+\beta_{L N T A} L N T A_{i, t}+\beta_{M K T} M K T_{t}+\varepsilon_{i, t+1}$. Standard errors in paranthesis. ${ }^{*}$ significant at $10 \%,{ }^{* *}$ significant at $5 \%, * * *$ significant at $1 \%$. 


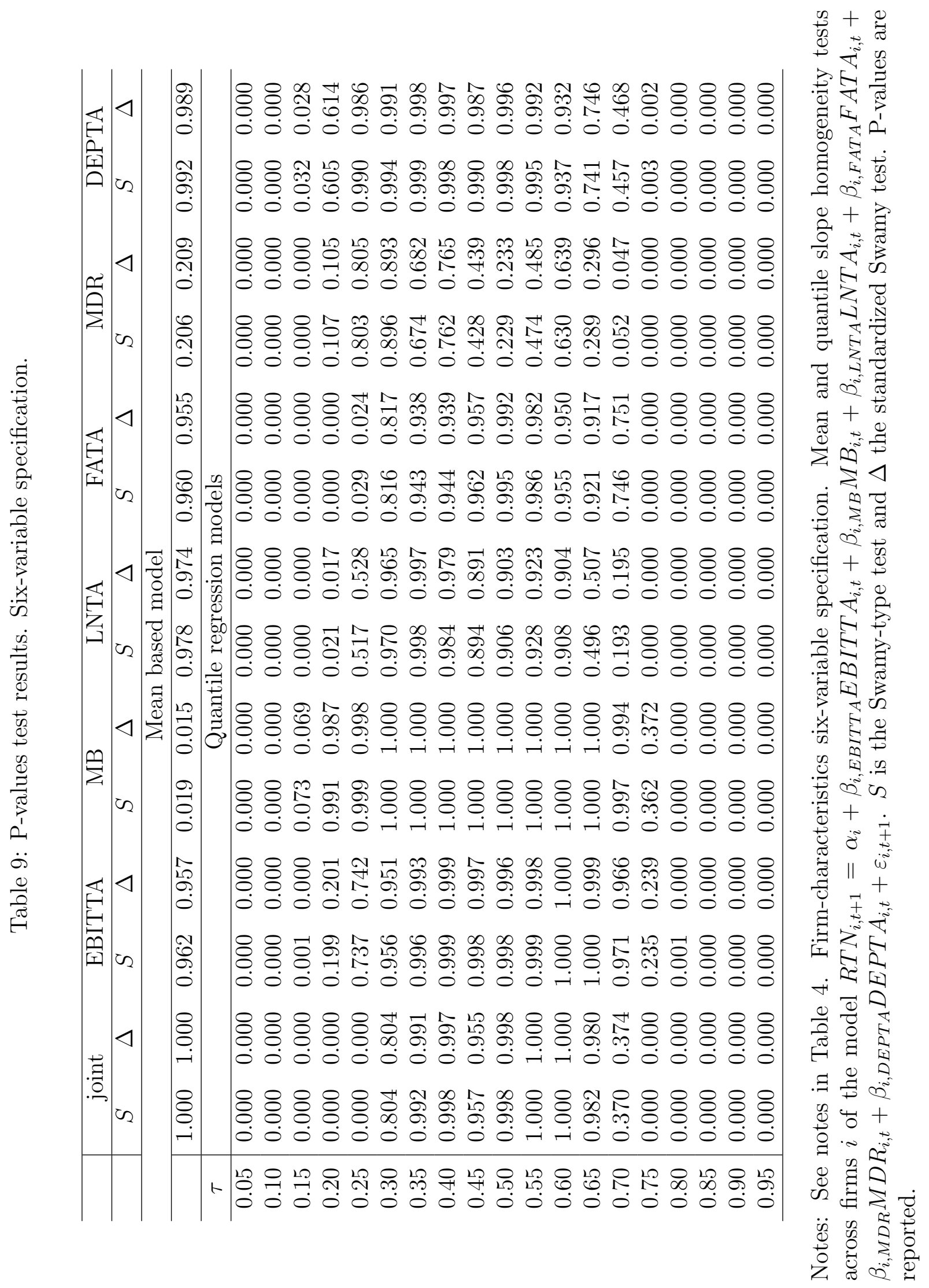


Table 10: FE and FE QR estimation results. Six-variable specification.

\begin{tabular}{c|cccccc} 
variables & mean & $\tau=0.10$ & $\tau=0.25$ & $\tau=0.50$ & $\tau=0.75$ & $\tau=0.90$ \\
\hline EBITTA & $-0.602^{* * *}$ & $-0.286^{* *}$ & $-0.510^{* * *}$ & $-0.530^{* * *}$ & $-0.615^{* * *}$ & $-0.539^{* * *}$ \\
& $(0.098)$ & $(0.113)$ & $(0.031)$ & $(0.000)$ & $(0.037)$ & $(0.171)$ \\
MB & $-0.042^{* * *}$ & $-0.046^{* * *}$ & $-0.035^{* * *}$ & $-0.041^{* * *}$ & $-0.020^{* * *}$ & 0.001 \\
& $(0.010)$ & $(0.011)$ & $(0.003)$ & $(0.000)$ & $(0.003)$ & $(0.013)$ \\
LNTA & $0.058^{* * *}$ & $0.137^{* * *}$ & $0.081^{* * *}$ & $0.051^{* * *}$ & $0.027^{* * *}$ & 0.007 \\
& $(0.010)$ & $(0.010)$ & $(0.003)$ & $(0.000)$ & $(0.003)$ & $(0.015)$ \\
FATA & $0.620^{* * *}$ & $1.178^{* * *}$ & $0.751^{* * *}$ & $0.480^{* * *}$ & $0.432^{* * *}$ & -0.104 \\
& $(0.091)$ & $(0.095)$ & $(0.029)$ & $(0.000)$ & $(0.033)$ & $(0.145)$ \\
MDR & $1.089^{* * *}$ & $1.076^{* * *}$ & $0.943^{* * *}$ & $0.894^{* * *}$ & $1.097^{* * *}$ & $1.293^{* * *}$ \\
& $(0.051)$ & $(0.057)$ & $(0.016)$ & $(0.000)$ & $(0.019)$ & $(0.083)$ \\
DEPTTA & $-5.112^{* * *}$ & $-4.265^{* * *}$ & $-3.633^{* * *}$ & $-3.626^{* * *}$ & $-5.053^{* * *}$ & $-5.064^{* * *}$ \\
& $(0.598)$ & $(0.602)$ & $(0.184)$ & $(0.000)$ & $(0.218)$ & $(0.993)$ \\
\hline
\end{tabular}

Notes: See notes in Table 4. Firm-characteristics seven-variable specification. Mean and quantile FE model $R T N_{i, t+1}=\alpha_{i}+\beta_{E B I T T A} E B I T T A_{i, t}+\beta_{M B} M B_{i, t}+\beta_{L N T A} L N T A_{i, t}+$ $\beta_{F A T A} F A T A_{i, t}+\beta_{M D R} M D R_{i, t}+\beta_{D E P T A} D E P T A_{i, t}+\varepsilon_{i, t+1}$. Standard errors in paranthesis. $*$ significant at $10 \%,{ }^{* *}$ significant at $5 \%,{ }^{* * *}$ significant at $1 \%$. 


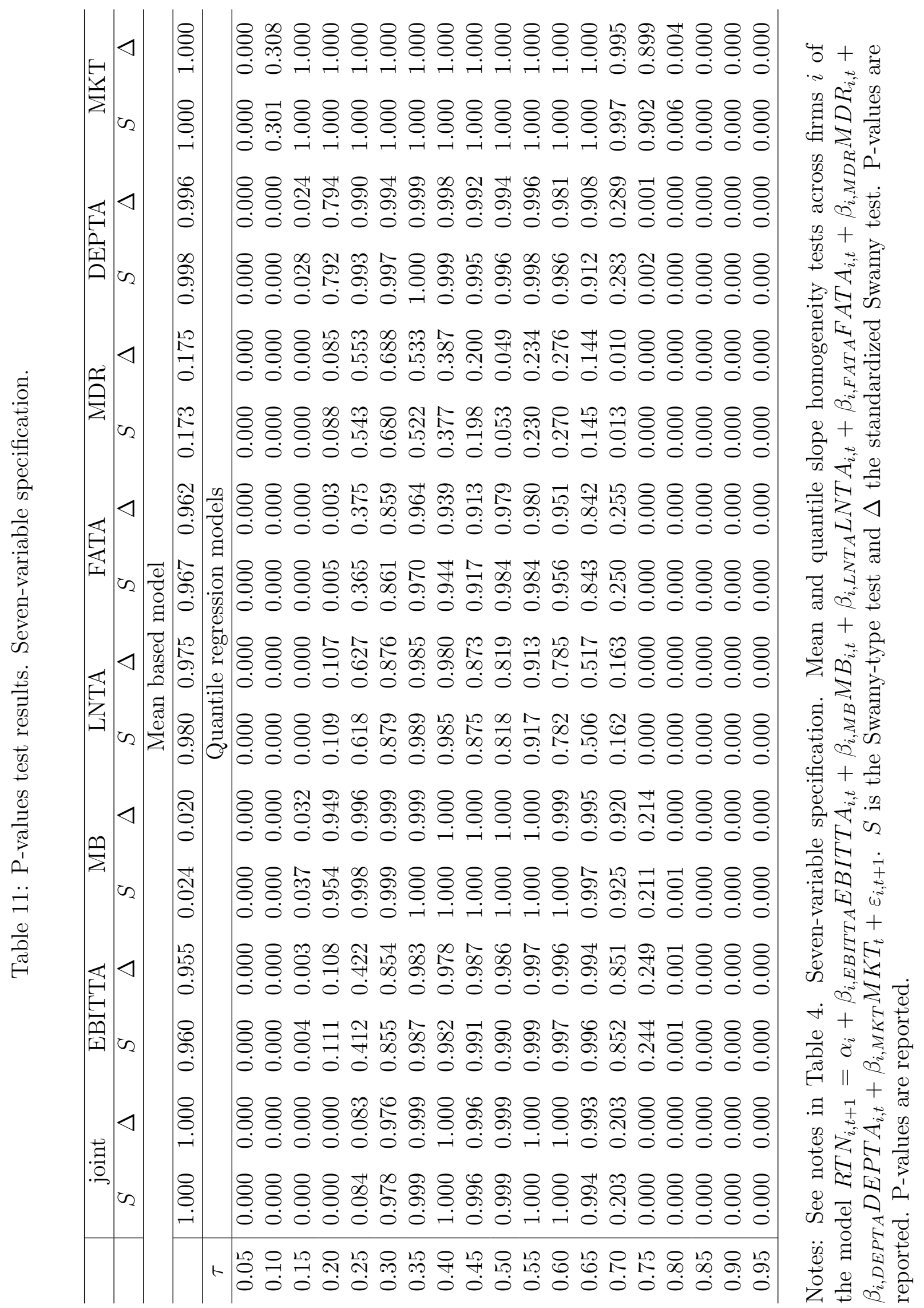


Table 12: FE and FE QR estimation results. Seven-variable specification.

\begin{tabular}{c|cccccc} 
variables & mean & $\tau=0.10$ & $\tau=0.25$ & $\tau=0.50$ & $\tau=0.75$ & $\tau=0.90$ \\
\hline EBITTA & $-0.602^{* * *}$ & $-0.286^{* *}$ & $-0.510^{* * *}$ & $-0.530^{* * *}$ & $-0.615^{* * *}$ & $-0.539^{* * *}$ \\
& $(0.098)$ & $(0.113)$ & $(0.031)$ & $(0.000)$ & $(0.037)$ & $(0.171)$ \\
MB & $-0.042^{* * *}$ & $-0.046^{* * *}$ & $-0.035^{* * *}$ & $-0.041^{* * *}$ & $-0.020^{* * *}$ & 0.001 \\
& $(0.010)$ & $(0.011)$ & $(0.003)$ & $(0.000)$ & $(0.003)$ & $(0.013)$ \\
LNTA & $0.058^{* * *}$ & $0.137^{* * *}$ & $0.081^{* * *}$ & $0.051^{* * *}$ & $0.027^{* * *}$ & 0.007 \\
& $(0.010)$ & $(0.010)$ & $(0.003)$ & $(0.000)$ & $(0.003)$ & $(0.015)$ \\
FATA & $0.620^{* * *}$ & $1.178^{* * *}$ & $0.751^{* * *}$ & $0.480^{* * *}$ & $0.432^{* * *}$ & -0.104 \\
& $(0.091)$ & $(0.095)$ & $(0.029)$ & $(0.000)$ & $(0.033)$ & $(0.145)$ \\
MDR & $1.089^{* * *}$ & $1.076^{* * *}$ & $0.943^{* * *}$ & $0.894^{* * *}$ & $1.097^{* * *}$ & $1.293^{* * *}$ \\
& $(0.051)$ & $(0.057)$ & $(0.016)$ & $(0.000)$ & $(0.019)$ & $(0.083)$ \\
DEPTA & $-5.112^{* * *}$ & $-4.265^{* * *}$ & $-3.633^{* * *}$ & $-3.626^{* * *}$ & $-5.053^{* * *}$ & $-5.064^{* * *}$ \\
& $(0.598)$ & $(0.602)$ & $(0.184)$ & $(0.000)$ & $(0.218)$ & $(0.993)$ \\
MKT & $0.002^{* * *}$ & $0.003^{* * *}$ & $0.002^{* * *}$ & $0.001^{* * *}$ & $0.0004^{* * *}$ & -0.0007 \\
& $(0.0004)$ & $(0.0005)$ & $(0.0002)$ & $(0.000)$ & $(0.0002)$ & $(0.0006)$ \\
\hline
\end{tabular}

Notes: See notes in Table 4. Firm-characteristics seven-variable specification. Mean and quantile FE model $R T N_{i, t+1}=\alpha_{i}+\beta_{E B I T T A} E B I T T A_{i, t}+\beta_{M B} M B_{i, t}+\beta_{L N T A} L N T A_{i, t}+$ $\beta_{F A T A} F A T A_{i, t}+\beta_{M D R} M D R_{i, t}+\beta_{D E P T A} D E P T A_{i, t}+\beta_{M K T} M K T_{t}+\varepsilon_{i, t+1}$. Standard errors in paranthesis. $*$ significant at $10 \%, * *$ significant at $5 \%, * * *$ significant at $1 \%$. 
Figure 1: Seven-variables specification, distribution of firm-specific coefficients
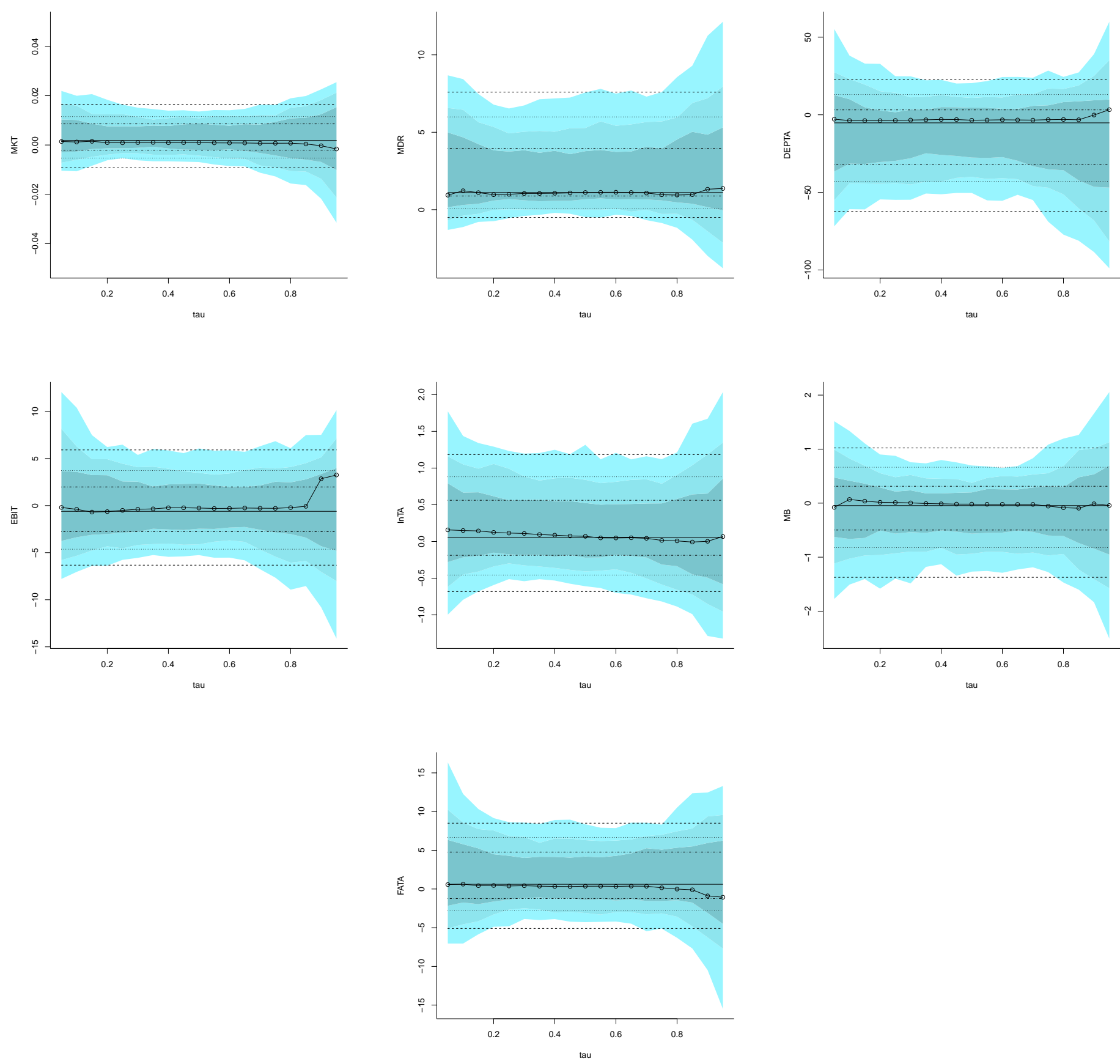

Notes: $0.05,0.10,0.20,0.80,0.90$ and 0.95 percentiles of the estimated firm-specific QR (for each $\tau$ ) and OLS coefficients. MD-OLS: solid line. MD-QR: solid line with circles. Mean and quantile regression firm-specific $i$ model:

$$
\begin{gathered}
R T N_{i, t+1}=\alpha_{i}+\beta_{i, E B I T T A} E B I T T A_{i, t}+\beta_{i, M B} M B_{i, t}+\beta_{i, L N T A} L N T A_{i, t}+\beta_{i, F A T A} F A T A_{i, t}+ \\
\beta_{i, M D R} M D R_{i, t}+\beta_{i, D E P T A} D E P T A_{i, t}+\beta_{i, M K T} M K T_{t}+\varepsilon_{i, t+1} .
\end{gathered}
$$

